\title{
Risk-Sharing and the Creation of Systemic Risk
}

\author{
Viral V. Acharya ${ }^{1}$, Aaditya M. Iyer ${ }^{2, *}$ and Rangarajan K. Sundaram ${ }^{1}$ \\ 1 Stern School of Business, New York University, 44 West Fourth Street, New York, NY 10012, USA; \\ vacharya@stern.nyu.edu (V.V.A.); rsundara@stern.nyu.edu (R.K.S.) \\ 2 Man Group, Man Investments Inc., 452 Fifth Avenue, 27th floor, New York, NY 10018, USA \\ * Correspondence: aiyer@stern.nyu.edu
}

Received: 24 June 2020; Accepted: 7 August 2020; Published: 17 August 2020

\begin{abstract}
We address the paradox that financial innovations aimed at risk-sharing appear to have made the world riskier. Financial innovations facilitate hedging idiosyncratic risks among agents; however, aggregate risks can be hedged only with liquid assets. When risk-sharing is primitive, agents self-hedge and hold more liquid assets; this buffers aggregate risks, resulting in few correlated failures compared to when there is greater risk sharing. We apply this insight to build a model of a clearinghouse to show that as risk-sharing improves, aggregate liquidity falls but correlated failures rise. Public liquidity injections, for example, in the form of a lender-of-last-resort can reduce this systemic risk ex post, but induce lower ex-ante levels of private liquidity, which can in turn aggravate welfare costs from such injections.
\end{abstract}

Keywords: banking; clearinghouses; systemic risk

JEL Classification: G21; G22; G31

\section{Introduction}

Over much of the last several decades, instruments and contracts aimed at facilitating risk-sharing between financial agents have seen explosive growth. By and large (although not uniformly) over this period, regulators viewed this as a benign and even beneficial development, a point of view perhaps best exemplified by Federal Reserve Board Chairman Alan Greenspan's remarks at the American Bankers Association Annual Convention in October 2004. ${ }^{1}$ Referring to the unbundling and transfers of risks facilitated by derivatives, Greenspan suggested that, as a consequence

... not only have individual financial institutions become less vulnerable to shocks from underlying risk factors, but also the financial system as a whole has become more resilient.

The advent of the financial crisis in 2007-2008 led to a sharp reappraisal of these views. Today, it is widely acknowledged that the very markets and contracts designed with the ostensible purpose of facilitating risk-sharing among financial intermediaries-including derivatives such as credit default swaps and securitization instruments such as collateralized debt obligations-played a central role in fanning the crisis, nearly bringing about a collapse of the entire financial system.

How could the facilitation of risk-sharing increase systemic risk and systemic fragility? In this paper, we suggest an explanation of this apparently paradoxical situation. The intuition behind our formal analysis is rather simply expressed. We begin with the observation that while risk-sharing arrangements are effective at hedging against idiosyncratic shocks (such shocks are by

1 http://www.federalreserve.gov/BOARDDOCS/Speeches/2004/20041005/default.htm (accessed on 17 July 2020). 
definition uncorrelated across agents), they have, at best, limited potential to provide a hedge against aggregate shocks. When an aggregate shock hits the economy, everyone is affected by the common shock and any existing risk-sharing arrangements face increased likelihood of counterparty default. The only effective ex-ante defense against systemic shocks is to, so to speak, save for the rainy day, viz., to increase holdings of safe, liquid assets ("cash") and reduce investment in risky and illiquid assets.

Now consider an agent who faces shocks that could be idiosyncratic or aggregate. Aggregate shocks are those that hit all agents and firms; they may be far less likely (they may be "tail risks") than the idiosyncratic risk exposures, and as such, the agent may find it privately costly to hold liquidity purely for withstanding such aggregate shocks. However, when risk-sharing opportunities are absent or scarce, the more-likely idiosyncratic shocks also need to be hedged, providing the agent with an incentive to hold liquid assets to withstand the shocks and making it privately optimal to save to hedge the risk exposures. As a consequence, in the (perhaps low-likelihood) event that an aggregate risk materializes, the system has adequate liquidity to deal with it, so systemic crises are averted or unlikely.

In contrast, when financial innovations allow agents and firms to share idiosyncratic risks with each other, the hedging motive for holding liquidity to withstand these shocks is reduced. Risk-sharing allows the financial system to economize on its pool of liquidity. Now, the principal benefit of holding liquidity arises in case of aggregate shocks, but if these are low-likelihood events, it will not generally be in the interest of individual agents or firms to hedge against these states. As a consequence, while idiosyncratic risks are shared and risk-sharing promises are honored when idiosyncratic shocks arise, there is inadequate liquidity and insufficient savings for the rainy day when an aggregate shock hits, making it difficult or impossible to honor risk-sharing promises. The result is financial fragility or systemic risk as an equilibrium outcome.

In short, financial innovations facilitating risk-sharing may increase systemic vulnerability by inducing agents to invest resources more fully in high-return risky projects by reducing liquidity for dealing with low-risk aggregate shocks. To be sure, the increased systemic fragility could be very costly for the economy when an aggregate shock arises since a meltdown of the financial sector could produce an elongated period of financial disruption affecting payment and settlement systems, transactional services, and savings schemes.

We deliver this insight in a series of models. Section 3 introduces the foundation on which we build, the problem of an individual firm seeking to hedge against total risk exposure in the absence of risk-sharing possibilities. (We call this no-risk-sharing setting one of "autarky.") We show that if the risk exposure is sufficiently large, the autarkic firm finds it privately optimal to fully hedge against the exposure by holding adequate liquidity. In Sections 4 and 5, we embed this one-firm setting in different multi-firm contexts (with each firm's risk exposure consequently broken into idiosyncratic and aggregate parts), and study the consequences of permitting risk-sharing among the firms.

Section 4 looks at the simplest and most transparent of these extensions, that of a two-firm setting in which the firms can write a risk-sharing contract to make transfers to each other in idiosyncratic risk states. When risk-sharing is disallowed, each firm is, of course, in the autarky setting of Section 3 , and for the reasons explained in the previous paragraph, there is adequate liquidity in the system to handle the aggregate risk state. However, when risk-sharing is allowed, we show that the firms optimally choose to economize on liquidity and increase their investment in risky assets; and, as a consequence, when the (possibly low-likelihood) aggregate risk state does materialize, the system-wide liquidity proves inadequate to meet the shock, and both firms fail.

Section 5 then generalizes the setting to a setting with a large number of firms and a richer description of uncertainty, and looks at risk-sharing via a co-insurance arrangement akin to a clearinghouse. The clearinghouse collects premiums up-front to build its reserves but it can further also make ex-post "capital calls" on liquidity-surplus banks to aid (co-insure) the liquidity-short firms. Clearinghouse insolvency and systemic failure occur if the reserves and capital calls are collectively insufficient to meet the needs of liquidity-short firms. In this case, a fire sale takes place and 
liquidity-surplus banks acquire the assets of liquidity-short banks. The fire sale is costly since banks do not realize full returns on acquired assets.

We show that in this case too our general insight prevails: risk-sharing arrangements can incentivize firms to take on greater risk at the individual level, reduce overall liquidity, and increase systemic fragility. The level of correlation in firm risks plays a key role. When the correlation between underlying risks of firms is low, risk-sharing arrangements are enabled that do not require high upfront premiums, and the clearinghouse setting provides risk-sharing of good quality in most states of the world, offering a clear benefit over a world without risk-sharing. However, when a large aggregate shock materializes, this arrangement fails, creating greater systemic fragility than in a world without risk-sharing. Conversely, when underlying risks of firms are highly correlated, upfront premiums required by insurance and clearinghouse rise correspondingly, reducing the attractiveness of risk-sharing. At sufficiently high correlations, premiums become so high that autarky is preferable and aggregate outcomes mirror the no risk-sharing setting.

While the clearinghouse leads to systemic risk, it also economizes ex-ante on liquidity held by banks. In other words, while there is increased risk of aggregate failure in the presence of a clearinghouse, the expected losses arising from this risk is countered by the expected gains to banks in making risky investments. This result breaks down, however, in the presence of a lender-of-last-resort (LOLR) that can choose to inject public liquidity into the clearinghouse at a cost. We model this extension in Section 6. While LOLR interventions lower the incidence of fire sales ex post, there is a countervailing ex-ante effect: banks internalize the LOLR intervention and hold even less private liquidity than is optimal under the first-best scenario. This increases welfare losses from LOLR interventions, even as fire sale costs are reduced, and creates the rationale for regulations such as minimum margin requirements on clearinghouse members.

Indeed, consistent with our model, Alessandri and Haldane (2009) document that capital ratios maintained by financial sectors in the United States and the United Kingdom have been declining steadily over time in the presence of a lender of last resort (the Federal Reserve Banks in the US and the Bank of England in the UK). This is illustrated in Figure 1.

\section{History of Banking Leverage in US and UK}

(Alessandri and Haldane, 2009)

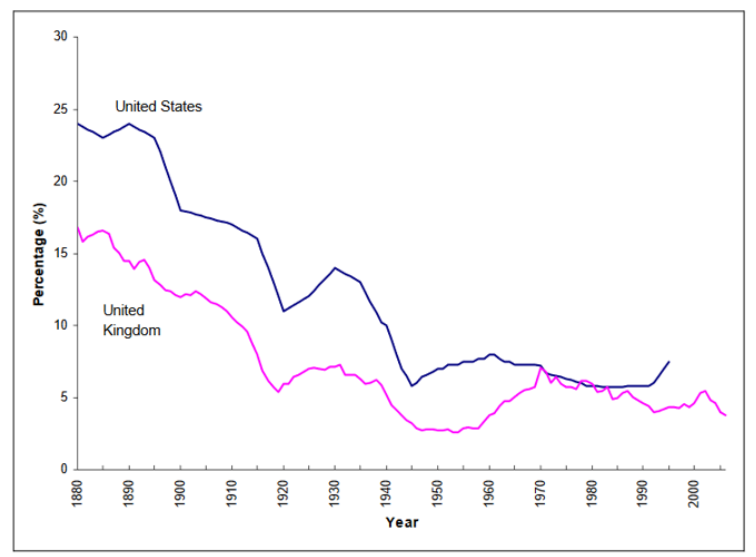
Source: US: Berger, A, Herring, R and Szegö, G (1995). UK: Sheppard, D.K (1971), BBA, published accounts

Figure 1. Bank capital ratios. This figure is taken from Chart 2 of Alessandri and Haldane (2009), and plots the evolution of capital ratios of US and UK banks over time. 
These models help illustrate our main point that aggregate risk translates into systemic risk or collective failures depending on the liquidity choices of agents which are endogenous to the available risk-sharing opportunities. ${ }^{2}$

The ideas in this paper may have general applicability beyond the immediate concerns analyzed here. Consider any setting in which much of the activity is "routine" but there is an occasional need to face a complex unexpected scenario. (Most organizations can, in fact, be described in this fashion as largely requiring a set of routine activities which in principle may be implemented following protocol or "rules," but occasionally an unexpected—and consequential—query arises that cannot be addressed within the rules. In our model, the routine is the management of idiosyncratic risk, the dramatic is that of systemic risk.) With primitive technology, the same resources are used to manage both sets of activities, the routine and the rare, so, as a consequence, there are generally adequate resources available to address crises when they arise. However, as technology improves facilitating specialized handling of activities, the management of the routine can be separated from the management of the unexpected; the consequent specialization reduces resources available to address the rare hit (it may no longer be economically viable to maintain resources just to address the rare complex event), and so makes the organization more vulnerable to derailment from tail events. Note, however, that while this is a general idea, this paper models and understands the implications of this idea in the context of risk-sharing between banks (and more generally financial intermediaries).

Section 2 discusses the related literature. Sections 3 and 4 contain our analysis for the cases of autarky and two banks respectively. Section 5 generalizes this framework to many banks forming a clearinghouse and discusses the characteristics of equilibria that occur, comparing them to first-best outcomes. Section 6 incorporates a lender of last resort in the clearinghouse model. Finally, Section 7 concludes. Proofs not contained in the main body of the paper may be found in the appendices.

\section{Related Literature}

As noted in the introduction, prior to the financial crisis, the view that risk-sharing and derivatives enhanced systemic stability was widely held, but there were important notes of dissent. Speaking in Jackson Hole, Wyoming, in August 2005, Rajan (2005), then Chief Economist of the IMF, commented that

While the system now exploits the risk-bearing capacity of the economy better by allocating risks more widely, it also takes on more risks than before. ... [T] The linkages between markets, and between markets and institutions, are more pronounced. While this helps diversify the system against small shocks, it also exposes the system to large systemic shocks. ${ }^{3}$

Rajan's (qualitative) case for why financial innovations may have made the world riskier focuses on tail-risk seeking that is driven by short-termism of financial sector pay and incentives. Our paper offers a formalization that is complementary and that leads to similar conclusions that the growth of markets for risk-sharing could result in greater risk-taking and make the system more vulnerable to "large systemic" shocks.

Acharya et al. (2010) document in detail the process of "manufacturing tail risks" that took hold during 2003-2007 and the reasons behind it, in particular, excessive seeking of aggregate risk due to the presence of government guarantees, imperfect regulation and its arbitrage, and inadequate internalization by the financial sector of externalities from collective failures. Our paper offers a viewpoint that is complementary to these. It suggests that improvements in financial risk-sharing technology and innovations, and the resulting moral-hazard effect on private liquidity choices,

2 As the models will make clear, we refer to aggregate risk as the risk arising from a bad realization of an aggregate shock that affects multiple banks and requires them to refinance. Systemic risk refers to the risk that multiple banks, that require refinancing, may simultaneously fail.

3 See Cassidy (2009, chp. 1, p. 21). 
may be the subtle underlying force behind the growing inadequacy of private reserves to withstand aggregate shocks. ${ }^{4}$

Several papers consider the effect of risk-sharing on risk-taking and are related to our paper. In an early contribution, Bhattacharya and Gale (1987) consider how inter-bank contracts to share liquidity can lead to free-riding on the common pool of liquidity as individual banks, privately informed about own liquidity shocks, aim to transfer risks to counterparties. Acemoglu and Zilibotti (1997) provide a model in which risk-averse agents when offered diversification opportunities are willing to take on risks that were privately too risky to undertake otherwise. Allen and Carletti (2006) model how ability to transfer risks from banking to the insurance sector can create risk of contagion from one sector to the other.

In more recent work on this theme, Acharya and Bisin (2009) consider how managerial incentives to take on aggregate or idiosyncratic risks are altered by the ability to privately hedge these risks by trading in capital markets. Zawadowski (2013) considers a market for insurance in an "entangled" financial network and shows how the presence of network externalities can lead in equilibrium to inefficiently little insurance purchase against low-risk events. Yorulmazer (2013) considers how the access to credit default swaps (insurance against default risk) can lead the insured and the insurer to "herd" as they collectively prefer correlated underlying risks to transfer risks outside of the financial sector, e.g., on to the regulator and the taxpayers.

While sharing some similarity with each of these papers linking risk-sharing to risk-taking, our primary insight is somewhat different in that ability to share risks alters the cost-benefit tradeoff in holding liquidity to hedge against aggregate risk states and this alteration can endogenously transform such states into collective failure or systemic risk states.

Finally, the moral hazard effect of risk-sharing that we propose and model provides a novel way of understanding the risks that are shared, and more importantly, risks that remain, from co-insurance arrangements such as clearinghouses.

Much of the existing literature on clearinghouses is focused on somewhat different issues: determining whether risks will be adequately pooled by single versus multiple clearinghouses (Duffie and Zhu 2011) and whether clearinghouses can resolve incomplete information about agents' positions (Leitner 2012; Acharya and Bisin 2014). Pirrong (2009) argues that costs relating to information asymmetry are higher with a clearinghouse and may potentially outweigh the benefits associated with the mutualization of risks. Menkveld (2017) identifies the systemic risk arising from crowded trades by clearinghouse members and proposes a margin methodology to account for it. In contrast, a recent speech by Tucker (2014) highlights the moral hazard consequences of clearinghouse arrangements and resonates with our analysis of clearinghouse arrangements (the creation of systemic risk relative to autarky).

There has been some recent work on the optimal design of clearinghouses and on the welfare implications of their introduction and use. Koeppl et al. (2012) consider the tradeoff faced by a clearinghouse in providing liquidity to members, while ensuring incentives are in place for carrying out and settling transactions. Paddrik and Zhang (2020) study the optimal default waterfall design to limit clearinghouse losses given a set of exposures. Biais et al. (2012) consider whether centralized clearing makes trading parties better off and whether it eliminates counterparty risk of its members. They build a model in which buyers of protection (who are risk-averse) trade with risk-neutral sellers who may default. To reduce this counterparty risk, buyers have an incentive to expend effort and trade with a "good" protection seller, with low counterparty risk. In line with our findings, the authors show that-if risks are idiosyncratic — the clearinghouse is able to fully insure agents against possible future

4 This, in turn, may also explain the explosive growth of the financial sector over the past two decades (Philippon and Reshef 2012) relative to other sectors of the economy; the growth occurred at the cost of grave risk of future crises whose costs were partly borne by sectors other than the financial sector. 
losses. If there is aggregate risk, however, agents expend effort to search for good counterparties, and are fully insured only in the case when effort is observable and contractible.

Our model, however, is different in that it focuses on the assumption of risk undertaken by every bank (and hence the aggregate) when risk sharing is and is not possible. We show that risk sharing endogenously incentivizes the agents in our model to take on more risk leading to an increased likelihood of systemic failure, and efficiency losses resulting from a fire sale. The fire sale is modeled along the same lines as in Acharya et al. (2011), who study losses associated with transfers of control from domestic to less efficient foreign firms in a crisis.

Rochet and Roger (2016) build a general contracting theory of "risky utilities"—firms that provide public benefits while still at the risk of default, such as clearinghouses, large banks and infrastructure companies. They show that the optimal regulatory contract is implemented with a capital requirement, which, if breached, results in restructuring and expropriation. In contrast, we show that a simple margin requirement can serve to increase welfare when clearinghouse members do not internalize the cost of systemic risk, and that this regulation is at its most effective when the correlation between the shocks to member banks is high.

Castiglionesi et al. (2017) argue how increased financial integration can lead to a less stable banking system in the presence of aggregate shocks. They apply this idea in the contet of the inter-bank lending market and show how interest rates can be more skewed in the presence of greater financial integration. By contrast, our paper applies this idea to clearinghouses, from which we obtain different policy implications. Additionally, our paper studies how the presence of a Lender of Last Resort can affect bank choices and welfare outcomes.

\section{The One-Bank Setting}

\subsection{Model and Payoffs}

In this section, we introduce the foundational one-bank model that we embed in multi-bank settings in Sections 4 and 5 . We consider a model with three dates $t \in\{0,1,2\}$. At date 0 , a bank has initial investable capital of $\$ 1$. Banks have access to two investment opportunities. The first is a safe asset ("cash"). The safe asset has a net return of zero; an investment of $\ell$ at time $t$ returns $\ell$ at time $t+1$.

The second investment opportunity is in a risky asset or risky project. Figure 2 summarizes the consequences of investing in this opportunity. Investment in this risky project at time 0 yields returns at time 2. The risk in the project comes from the potential need to refinance the investment at time 1. There is a probability $\alpha$ that such refinancing will be required. Refinancing is a zero-one decision; partial refinancing of the project is not possible. The refinancing amount required is $\$ \varphi$ per $\$ 1$ that was invested in the project. For specificity, we take $\varphi=1$; i.e., if refinancing is required, the refinancing need is $100 \%$ of the initial invested amount in the risky project. ${ }^{5}$

If the project does not require refinancing at time 1 , or if it requires refinancing and is refinanced, then the expected return at time 2 is $R>1$ per unit investment in the project at time 0 . If refinancing is needed and the project is not refinanced, the investment returns zero with certainty at time 2 .

5 This is for expository purposes to more easily illustrate our main point that increased risk sharing can lead to greater systemic risk. Appendix B contains a more detailed discussion on bank behavior given a more general value of $\varphi$ for both the 1-bank and the 2-bank model. Our results-that there exists a risk-sharing equilibrium that leads to greater systemic risk than in autarky—continue to hold. 


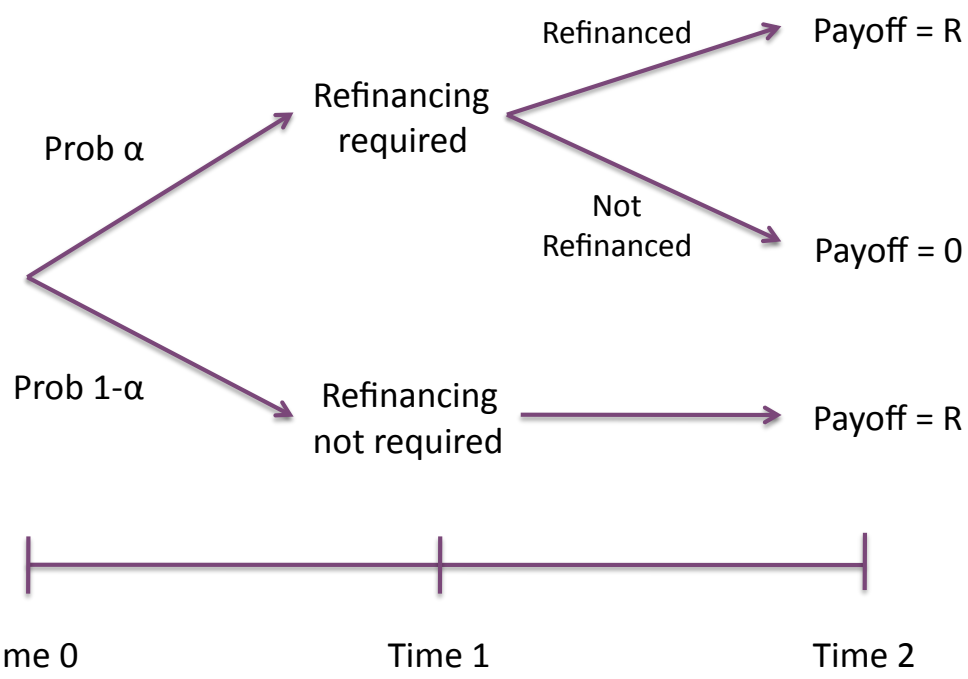

Figure 2. The risky investment opportunity. This figure summarizes the salient features of the risky investment opportunity described in Section 3.

Since $R>1$, it is always worth refinancing the project at time 1 should such refinancing be required (and should it be feasible). We make two assumptions concerning the refinancing. First, we assume that the refinancing need is observable and verifiable. This assumption plays no role in the current section, but is used in a later section when we consider allowing the bank to use a contingent claim to guard against this state. Second, we assume that the cash flows from the risky project are not pledgeable, so the bank cannot raise outside financing against these cash flows. For the time being (until we introduce risk-sharing possibilities), this means the project may only be refinanced using the bank's own internal resources, which in turn means it can only be refinanced using the amount the bank has invested in the riskless asset.

\subsection{The Bank's Optimal Investment Decision}

The tradeoff driving the bank's investment decision is a simple one: the riskless asset's return is lower than that from the risky project, but investing (a suitable amount) in the riskless asset provides the bank with the ability to refinance the project should refinancing be required-which happens with probability $\alpha$. We show that if $\alpha$ is small, then it is not worthwhile for the bank to divert investment away from the risky project; there is no investment in the riskless asset and the risky project fails with probability $\alpha$. As $\alpha$ increases past a critical level, then refinancing becomes optimal; the bank fully self-insures and invests enough in the riskless asset to meet the refinancing need (should it arise), and the risky project never fails. Finally, at very high levels of $\alpha$, investment in the risky project becomes unprofitable and all investment is made in the riskless asset.

Specifically, let $(\ell, 1-\ell)$ denote the investment strategy in which the bank chooses to invest $\ell$ in the riskless asset and $1-\ell$ in the risky technology. We prove the following result:

Proposition 1. Let $\lambda(R)$ and $v(R)$ be defined by

$$
\lambda(R)=\frac{R-1}{2 R-1} ; \quad v(R)=R-1 .
$$

Then:

1. If $\alpha \in[0, \lambda(R))$, then the optimal action is $\ell^{*}=0$. Since there is no investment in the riskless asset, the risky project is never refinanced so fails with probability $\alpha$. The bank's expected payoff is $(1-\alpha) R$. 
2. If $\alpha \in[\lambda(R), v(R)]$, the optimal action is $\ell^{*}=1 / 2$. There is always exactly enough to refinance the risky project if this is required. The risky project is always refinanced, so never fails. The bank's expected payoff is

$$
\frac{1}{2}[R+1-\alpha]
$$

3. If $\alpha>v(R)$, the optimal action is $\ell^{*}=1$. There is no investment in the risky project and the bank's expected payoff is 1 .

Remark 1. Note that Case 3 is irrelevant when $R \geq 2$ since $\alpha \leq v(R)$ is always satisfied in this case.

Proof. See Appendix A.

In the sections following we extend this model in several directions. Section 4 considers a basic extension to a two-bank setting and looks at the consequences of permitting risk sharing in this set-up. Section 5 develops a many-firms model and look at the consequences of permitting risk-sharing via a third-party insurance-style arrangement and a clearinghouse structure, respectively.

\section{Two-Banks: Risk-Sharing and Increasing Systemic Risk}

Consider a two-bank setting in which each bank faces exactly the same investment opportunities as in the previous section with exactly the same payoffs, but in which the refinancing needs may be correlated. Thus, there are four states of the world that are possible:

\begin{tabular}{ccl}
\hline State & Probability & Risky Projects Outcome \\
\hline 1 & $p_{1}$ & Neither project requires refinancing \\
2 & $p_{2}$ & Only Bank 2 needs refinancing \\
3 & $p_{3}$ & Only Bank 1 needs refinancing \\
4 & $p_{4}$ & Both banks need refinancing \\
\hline
\end{tabular}

We refer to States 2 and 3 as the "idiosyncratic" risk states and to State 4 as the one of "aggregate" or "systemic" risk. The total probability of Bank 1 needing refinancing $\left(p_{3}+p_{4}\right)$ and the total probability of Bank 2 needing refinancing $\left(p_{2}+p_{4}\right)$ are each taken to equal $\alpha$, where $\alpha$ is the probability in the one-bank setting of a refinancing need. For an arbitrary correlation $\rho$ in refinancing needs, the probabilities $p_{i}$ take on the form ${ }^{6}$

$$
\begin{aligned}
& p_{1}=\rho(1-\alpha)+(1-\rho)(1-\alpha)^{2} \\
& p_{2}=(1-\rho) \alpha(1-\alpha) \\
& p_{3}=(1-\rho) \alpha(1-\alpha) \\
& p_{4}=\rho \alpha(1-\alpha)+\alpha^{2}
\end{aligned}
$$

Note that the banks are ex-ante identical. We focus on the case $\alpha \in[\lambda(R), v(R)]$. As noted in Proposition 1, the bank under autarky then invests in the risky project but fully (self-)insures this investment so there is no failure.

We allow the banks to "risk-share" by trading in contingent claims that pay off in specified states of the world. When contingent claim trading is disallowed, each bank faces precisely the one-bank model of Section 3 the solution to which is identified in Proposition 1; we refer to this as the model of "autarky." Of course, even if contingent claim trading is allowed, each bank has the option to not

6 These probabilities can be derived by using the properties of Bernoulli random variables. For example, if $Z_{1}$ is an indicator variable equal to 1 if Bank 1 fails, and $Z_{2}$ is an indicator variable equal to 1 if bank 2 fails, then the probability that both banks fail is just $E\left(Z_{1} Z_{2}\right)=\operatorname{Cov}\left(Z_{1}, Z_{2}\right)+E\left(Z_{1}\right) E\left(Z_{2}\right) . E\left(Z_{1}\right)=E\left(Z_{2}\right)=\alpha$ and $\operatorname{Cov}\left(Z_{1}, Z_{2}\right)=\rho \alpha(1-\alpha)$, from which we get the results. The other probabilities are computed similarly. 
participate in the contingent claim market; it will elect to participate if and only if participation raises its expected payoff beyond that in the autarkic solution.

Risk-sharing affords each bank the possibility of lowering its investment in the safe asset while increasing its investment in the risky asset and relying instead on additional funds from the contingent claims should refinancing of its risky project be required. There is a chance, of course, that the refinancing shock may be "systemic," i.e., both banks may require refinancing and there may not be enough cash to meet the combined refinancing needs. ${ }^{7}$ Thus risk-sharing involves a trade-off between the larger returns obtained from investing in the risky project and its failure in the systemic state.

Observe that risk-sharing benefits accrue in the idiosyncratic-risk States 2 and 3 but not in the systemic-risk State 4 . The likelihood of the idiosyncratic-risk states decreases, and that of the systemic-risk state increases, as the correlation $\rho$ in refinancing needs increases. Intuitively, therefore, one would expect that there is a critical cut-off level of $\rho$ below which risk-sharing may dominate the autarkic outcome but above which autarky should dominate. Proposition 2 establishes precisely this to be the case:

Proposition 2. Fix any $R>1$. There is a critical level of correlation $\rho^{*}>0$ given by

$$
\rho^{*}=\frac{1}{2 R}
$$

such that

1. For $\rho<\rho^{*}$ :

(a) There is an interval of values $\left[\lambda(R), \alpha^{*}\right)$ of $\alpha$ under any of which there is a risk-sharing equilibrium in which each bank invests an amount $2 / 3$ in the risky project and an amount $1 / 3$ in the riskless asset.

(b) Outcomes in this risk-sharing equilibrium strictly dominate outcomes under autarky.

(c) There is a positive probability given by $\rho \alpha(1-\alpha)+\alpha^{2}$ in this risk-sharing equilibrium of systemic failure (i.e., of both projects needing refinancing and neither obtaining it).

2. For $\rho>\rho^{*}$, the outcomes under autarky dominate those under risk-sharing.

Proof. See Appendix B.

The risk-sharing equilibrium described in Proposition 2 has the following properties:

1. There is greater individual risk-taking in the form of investment in the risky project by each bank under risk-sharing than under autarky (2/3 compared to $1 / 2)$, and correspondingly lower total liquidity/cash reserves held in the system under risk-sharing than under autarky ( $2 / 3$ compared to 1 ).

2. A systemic failure (State 4 with no accompanying refinancing) happens with positive probability in the risk-sharing equilibrium whereas, for the same parameters, there is no systemic failure under autarky; indeed, there are no individual failures under autarky because each bank maintains adequate reserves to insure itself.

3. Thus, expected systemic shortfall is zero under autarky but, under risk-sharing, each bank falls $2 / 3$ short of its refinancing need in the systemic failure state, so expected systemic shortfall is equal to

$$
2 \times \frac{2}{3} \times\left(\rho \alpha(1-\alpha)+\alpha^{2}\right)=\frac{4}{3}\left(\rho \alpha(1-\alpha)+\alpha^{2}\right) .
$$

7 Note that if there was enough cash to meet combined refinancing need, each bank must have invested enough in the riskless asset to meet at least its own refinancing need, so we are effectively in the autarky solution. 
The results of this section have been derived in a setting that is simple and transparent but also obviously restrictive (only two firms sharing risks, etc.). In the sections that follow, we describe a richer framework and examine the consequences of risk-sharing in a clearinghouse-style co-insurance arrangement (Section 5).

A final comment is relevant. The results of this section do not, in themselves, carry any welfare implications, in particular, that risk-sharing is "bad" in the sense of being welfare-decreasing. Indeed, if there are no externalities from systemic failures, then welfare is (trivially) increased by allowing for risk-sharing. However, if there are social and macroeconomic costs of bank bankruptcy and systemic failure (resulting, for example, from disruptions in the payment system), risk-sharing may well be welfare-reducing also.

\section{Clearinghouse: A Co-Insurance Model}

In this section, we move to a setting with a large number of banks and a richer description of uncertainty. Refinancing needs are driven by a common factor and also by idiosyncratic factors. When viewed in isolation, each individual bank in this model continues to face the same decision problem as in the one-bank setting of Section 3; thus, if the bank decides to remain in autarky and not participate in risk-sharing, its optimal actions are those specified by Proposition 1.

\subsection{Description of Uncertainty}

Suppose there are $n$ banks. If Bank $i$ invests an amount $y>0$ in the risky project, it receives an interim signal $A_{i} \sim \Phi(0,1)$ concerning the returns from the project, where $\Phi(\cdot)$ denotes the cumulative standard normal distribution. If $A_{i} \leq c_{i}$ (where $c_{i}$ is a given critical cut-off), then the project needs to be refinanced, i.e., an additional amount $y$ needs to be invested in the project. If the project is not refinanced, it returns zero with certainty. If it is refinanced, it returns $R>1$ with certainty.

The banks are ex-ante identical, and in particular $c_{1}=\cdots=c_{n}\left(=c\right.$, say). We let $\alpha=\operatorname{Prob}\left(A_{i} \leq\right.$ $c)=\Phi(c)$ be the ex-ante probability that a bank will need refinancing. We assume that the signals $A_{i}$ satisfy $^{8}$

$$
A_{i}=\sqrt{\rho} A+\sqrt{1-\rho} \epsilon_{i}
$$

where $A, \epsilon_{i} \sim \Phi(0,1)$, and $A$ and the $\epsilon_{i}$ 's are independent. Under this condition, banks' refinancing needs have a pairwise correlation of $\rho \in(0,1)$. We refer to $A$ as the common or aggregate factor in determining refinancing needs and to the $\epsilon_{i}{ }^{\prime}$ s as bank-specific or idiosyncratic factors. Conditional on $A=a$, note that the probability $p(a)$ that a generic bank needs refinancing is

$$
\begin{aligned}
p(a) & =\operatorname{Prob}\left\{\sqrt{\rho} a+\sqrt{1-\rho} \epsilon_{i} \leq c\right\} \\
& =\operatorname{Prob}\left\{\epsilon_{i} \leq \frac{c-\sqrt{\rho} a}{\sqrt{1-\rho}}\right\}=\Phi\left(\frac{c-\sqrt{\rho} a}{\sqrt{1-\rho}}\right) .
\end{aligned}
$$

We assume that there is a "clearinghouse" wholly owned by the banks that provides insurance to member banks from counterparty credit risk. The clearinghouse is funded with up-front premium or "margin" payments of size $k$ from each of the participating banks; in addition, the clearinghouse also has the right to make ex-post capital calls on its members. Specifically, in period 1, when banks' refinancing needs are known, these are first funded out of the margins collected by the clearinghouse and liquidity carried over by all member banks. Should this prove insufficient, the clearinghouse can then pledge a fraction $\tau$ of future payoffs realized by the investments of those banks that did not experience a refinancing need. However, if the initial margins and these capital calls prove collectively inadequate to meet the total refinancing needs, then the clearinghouse becomes insolvent and no bank's refinancing insurance contract is honored. In this event, we assume that the clearinghouse 
uses its margins to refinance as many banks as possible. We also assume that the refinancing choice is made randomly, i.e., ex-ante, every insolvent bank has the same probability of being refinanced by the clearinghouse. Those banks which have not been refinanced will sell their assets to solvent banks in a fire sale that we describe later in this section.

Banks take into account both the possibility of ex-post transfers and the chance of participating in the fire sale in their decision to deposit margin in the clearinghouse. Banks choose the level of margin they contribute to the clearinghouse, as well as the liquidity they choose to carry over for purchasing assets in the fire sale in case they remain solvent.

Conditional on insurance remaining solvent, the size of the transfers from the successful banks to the failed banks is a function of the fraction of banks requiring refinancing. This fraction (or more accurately, the distribution of this fraction) depends on the size of the aggregate shock. We look to characterize the equilibria that result in this setting.

As mentioned, $k$ denotes the up-front margin paid by each bank to the clearinghouse. Let $\ell$ denote the liquidity carried by each bank. Per dollar invested in the risky project, the bank obtains a return of $R$ if it is successful on the investment. Each bank is subject to an aggregate shock $a$ as well as an idiosyncratic shock $\epsilon_{i}$ and needs to refinance if $\sqrt{\rho} a+\sqrt{1-\rho} \epsilon_{i}<c$. Banks are ex-ante identical. Conditional on the aggregate shock $a$, let $f(a)$ be the fraction of banks requiring refinancing. For expositional simplicity, we write $f$ for $f(a)$ in the steps that follow.

Conditional on the aggregate shock $a$, the aggregate revenue of the banks not requiring refinancing is $R(1-f)(1-k-\ell)$, while the total refinancing need is $f(1-k-\ell)$. Given the initial margin collection of $k$, and the initial liquidity holdings of $\ell$, this leaves a deficit of $f(1-k-\ell)-k-\ell$ to be shared between those banks that were successful, i.e., did not experience a refinancing need. Recall that the clearinghouse can pledge a fraction $\tau$ of solvent banks' payoff (if required) to refinance those that have failed. Thus, the share $\eta(f)$ of its realized revenues that each successful bank must give up to the clearinghouse is

$$
\eta(f)=\frac{f(1-k-\ell)-k-\ell}{\tau R(1-k-\ell)(1-f)}
$$

If $\eta(f)>1$, then the clearinghouse cannot meet the refinancing needs even with the ex-post capital calls, so becomes insolvent. Note that we may also have $\eta(f)<0$; this simply means that the clearinghouse has a surplus left over after meeting refinancing needs and returns this amount to the successful banks.

Therefore, we now have two cases:

- $\quad f>[\tau R(1-k-\ell)+k+\ell] /[(1-k-\ell)(1+\tau R)]$, i.e., $\eta(f)>1$ : The clearinghouse fails and uses its margin $k$ to refinance a fraction of the failed banks. The remaining insolvent banks sell their assets in a fire sale. Solvent banks do not need refinancing and realize the return on their risky investment. They also purchase assets if a fire sale takes place.

- $f \leq[\tau R(1-k-\ell)+k+\ell] /[(1-k-\ell)(1+\tau R)]$ : Failed banks have their refinancing needs honored, realizing (after the bailout) a payoff of $R(1-k-\ell)$. Successful banks also lose a fraction $\eta(f)$ of the pledgeable portion of their payoff to fund the bailout.

Recall that

$$
f=f(a)=\Phi\left(\frac{c-\sqrt{\rho} a}{\sqrt{1-\rho}}\right) .
$$

Let $a_{0}(k, \ell)$ be such that $f\left(a_{0}(k, \ell)\right)=[\tau R(1-k-\ell)+k+\ell] /[(1-k-\ell)(1+\tau R)]$. Then the clearinghouse fails and a fire sale takes place if $a<a_{0}(k, \ell)$, and is solvent for $a \geq a_{0}(k, \ell)$.

The next subsections outline the fire sale process before characterizing the equilibrium.

\subsection{The Fire Sale}

We will assume that the fire sale of firms' assets takes place according to the following rules: 
(1) Each solvent bank takes prices as given, and submits a demand function to acquire assets—which are perfectly divisible- of the insolvent banks.

(2) Given the price, demand for each bank is constrained by the amount of liquidity it has at time 1 .

(3) The initial margin the clearinghouse collects, $k$, is used to bail out some of the firms that need refinancing.

(4) There is full liquidation of failed firms' assets. So the payoff for an insolvent bank is just the price at which it sells its assets, along with any liquidity it may carry over into time 1.

(5) Solvent banks can pledge a fraction $\tau$ of payoffs to acquire assets. These banks can only generate a return of $R-\Delta$ from acquired assets.

Let us assume that the clearinghouse has failed and that there will be a fire sale of assets of the insolvent firms. As before, let $f(a)$ be the number of firms that have failed, where $a$ is the value of the aggregate shock. Recall that

$$
f(a)=\Phi((c-\sqrt{\rho} a) / \sqrt{1-\rho})
$$

where $\Phi(\cdot)$ is the CDF of the shocks.

The clearinghouse fails and the fire sale takes place when

$$
f(a)>f\left(a_{0}(k, \ell)\right)=\frac{\tau R(1-k-\ell)+k+\ell}{(\tau R+1)(1-k-\ell)}
$$

Let the number of firms refinanced by the clearinghouse be given by $g(k, \ell)$, where $k$ is the clearinghouse margin and $\ell$ is the liquidity carried over by each bank from time 0 . Since each failed bank requires $(1-k-\ell)$ to refinance, we have that $g(k, \ell)=k /(1-k-\ell)$.

The number of banks selling assets in the fire sale equals $f(a)-g(k, \ell)$ while the number of banks buying assets equals $1-f(a)$, if bailed out banks do not participate in the fire sale. Let $y(p, k, \ell)$ denote the demand function submitted by each acquiring bank in the fire sale.

The market clearing condition is given by

$$
y(p, k, \ell)[1-f(a)]=(1-k-\ell)[f(a)-g(k, \ell)]
$$

The demand curves also satisfy

$$
y(p, k, \ell)=\frac{(\ell+\tau R(1-k-\ell)-y(p, k, \ell))^{+}}{p} \text { where } x^{+}=\max (x, 0)
$$

To understand the above expression, note that-given a price $p$ - the firm has liquidity it carries over, together with the payoff realized at time 1 . These realized payoffs equal a fraction $\tau$ of future payoffs given by $R(1-k-\ell)$. The firm also has to refinance projects it acquires, however, and has to pay a unit amount per asset acquired.

The fire sale price is therefore given by

$$
p(k, \ell)=\max \left(0,-1+[\ell+\tau R(1-k-\ell)] \frac{(1-f(a))}{(1-k-\ell)(f(a)-g(k, \ell))}\right)
$$

Acquiring banks profit from the fire sale when $p$ is less than $(R-\Delta-1)$. This corresponds to the condition $f>f_{1}(a)$ where $f_{1}$ is given by

$$
f_{1}(k, \ell)=\frac{(\ell+\tau R(1-k-\ell))+k(R-\Delta)}{\ell+\tau R(1-k-\ell)+(1-k-\ell)(R-\Delta)}
$$


For $f>f_{1}, p<(R-\Delta-1)$. In addition, to prevent prices being negative, we must have that $p=0$ when $f>\underline{f}(k, \ell)$, where

$$
\underline{f}(k, \ell)=\frac{k+\bar{\ell}+\tau R(1-k-\ell \overline{)})}{(1-k-\bar{\ell})+(\bar{\ell}+\tau R(1-k-\bar{\ell}))}
$$

Note that the fire sale only happens when insolvent banks cannot refinance themselves, i.e., $\ell<(1-k-\ell)$. If $\ell$ were greater than $(1-k-\ell)$, then banks would always choose to refinance since the gains from refinancing and obtaining the return of $R$ on risky assets would always exceed the payoff from selling assets in the fire sale and a price lower than or equal to $(R-\Delta-1)$.

\subsection{Prices and Profits}

In this table, we summarize the model in the above section and explicitly list the fire sale prices and the demand for each bank in the fire sale. Insolvent firms obtain the proceeds from the fire sale as payoff. This payoff equals the fire sale price multiplied by the assets requiring refinancing. Solvent firms get the payoff they generate from acquired assets, $(R-\Delta-1)$, net of fire sale price, as profit per unit of asset acquired in the fire sale. The total profit for acquiring banks is listed in the fourth column of the table. For simplicity, let us denote $f\left(a_{0}(k, \ell)\right)$ by $f_{0}$. Recall that a fire sale happens only when the clearinghouse becomes insolvent and $f>f^{*}$

\begin{tabular}{cccc} 
Region & Fire sale price & Fire sale demand & Profits (for acquiring firms) \\
\hline$f \in\left[f_{0}, f_{1}\right)$ & $(R-\Delta-1)$ & $y(R-\Delta-1, k, \ell)$ & 0 \\
$f \in\left[f_{1}, f\right]$ & $p(k, \ell)$ & $y(p, k, \ell)$ & $(R-\Delta-1-p) y(p, k, \ell)$ \\
$f \in[\underline{f}, \overline{1}]$ & 0 & $y(0, k, \ell)$ & $(R-\Delta-1) y(0, k, \ell)$
\end{tabular}

Let $a_{1}$ and $\underline{a}$ denote the corresponding value of the aggregate shock if the number of failures equals $f_{1}$ and $f$ respectively. Then, from the above table, we see that for surviving firms, profit from the fire sale is 0 if $\bar{a}>a_{1}$, while insolvent firms get a price $(R-\Delta-1)$ for their assets. Since each insolvent firm sells $(1-k-\ell)$ of assets in the fire sale, its profit from the fire sale is just $(1-k-\ell) \times p$, where $p$ is the fire sale price. Further, insolvent firms carry over the liquidity, $\ell$ from time 0 . If $a>a_{0}(k, \ell)$, the clearinghouse is solvent. There are no fire sales and the profit is given by total payoff net of transfer $\eta(k, \ell)$.

When computing expected profits for a given bank, we will assume that the clearinghouse sets the margin $k$, and all the other banks carry over liquidity $\bar{\ell}$. The bank takes $\bar{\ell}$ as given, and chooses $\ell$ accordingly. We will derive profits as a function of $k, \ell$ and $\bar{\ell}$.

Since all other banks carry over $\bar{\ell}$, and since each bank is atomistic, economy-wide variables such as prices, thresholds and transfers will be given by $\bar{\ell}$ instead of $\ell$. In particular, the fire sale price is given by $p(k, \bar{\ell})$. Clearing house failure is determined by the threshold $a_{0}(k, \bar{\ell})$, while transfers (in the case that the clearinghouse is solvent) is given by

$$
\eta(f)=\frac{f(1-k-\bar{\ell})-k-\bar{\ell}}{\tau R(1-k-\bar{\ell})(1-f)}
$$

Therefore, the payoff of a bank if the clearinghouse is solvent and if it carries liquidity $\ell$ is $\tau R[1-k-$ $\ell][1-\eta(f)]+(1-\tau R)(1-k-\ell)+\ell$ if it does not require refinancing, and $R[1-k-\ell]+\ell$ otherwise.

For notational ease, let $\underline{m}=\min \left(\underline{a}, a_{0}\right)$ and $m_{1}=\min \left(a_{0}, a_{1}\right)$.

Integrating over $a$, the ex-ante expected profit of a representative bank as a function of $k$ and $\ell$ is, therefore (where $\phi(\cdot)$ is the PDF of aggregate shock $a$ ): 


$$
\begin{aligned}
\mathbb{E} \Pi(k, \ell, \bar{\ell})= & \\
& \int_{-\infty}^{a_{0}(k, \bar{\ell})}[R(1-k-\ell)+\ell](1-f(a)+g(k, \bar{\ell})) \phi(a) d a \\
& +\int_{-\infty}^{\underline{m}}[(R-\Delta-1) y(0, k, \ell)(1-f(a))+\ell(f(a)-g(k, \ell))] \phi(a) d a \\
& +\int_{\underline{m}}^{m_{1}}[(R-\Delta-1-p) y(p, k, \ell)(1-f(a))+[p \times(1-k-\ell)+\ell](f(a)-g(k, \bar{\ell}))] \phi(a) d a \\
& +\int_{m_{1}}^{a_{0}(k, \bar{\ell})}[(R-\Delta-1)(1-k-\ell)+\ell)(f(a)-g(k, \bar{\ell})] \phi(a) d a \\
& +\int_{a_{0}(k, \bar{\ell})}^{\infty}([(\tau R(1-\eta(f(a)))+(1-\tau R))(1-f(a))+R f(a)](1-k-\ell)) \phi(a) d a
\end{aligned}
$$

The initial margin $k$ is chosen by the mutually-owned clearinghouse to maximize this profit. The choice of $k$ will evidently depend on the correlation $\rho$ and other factors. We look to characterize the optimal outcome, focusing in particular on the questions of whether risk-sharing can dominate autarky; whether risk-sharing leads to greater individual risk-taking than autarky; and whether risk-sharing leads to greater systemic failure (here insolvency of the clearinghouse) than autarky.

\subsection{Properties of Equilibrium}

As mentioned before, when solving for the equilibrium, we assume that the clearinghouse sets the margin level, $k$ paid by each bank. Banks can then choose liquidity $\ell$ to carry over from time 0 . When making this choice, banks take as given the liquidity carried over by the other banks. More formally, the equilibrium quantities $k^{*}$ and $\ell^{*}$ solve the following system:

$$
\begin{aligned}
k^{*} & =\underset{k}{\arg \max } \mathbb{E} \Pi\left(k, \ell^{*}(k)\right) \text { where } \\
\ell^{*}(k) & =\underset{\ell}{\arg \max } \mathbb{E} \Pi(k, \ell, \bar{\ell}) \text { and } \ell^{*}=\bar{\ell}
\end{aligned}
$$

The above equation states that banks choose liquidity $\ell^{*}$ taking as given liquidity $\bar{\ell}$ carried over by other banks (which affects the probability of failure of the clearinghouse). In a symmetric equilibrium, $\ell^{*}=\bar{\ell}$.

Characterizing the Equilibrium

It is clear from the expression earlier that expected profits are linear in $\ell$, i.e., expected profits can be written as

$$
\mathbb{E} \Pi(k, \ell, \bar{\ell})=\alpha_{0}(k, \bar{\ell})+\alpha_{1}(k, \bar{\ell}) \ell
$$

Since profit is linear in $\ell$, there is a bang-bang solution to the bank's choice of $\ell$. Therefore we have three cases:

Case 1: $\alpha_{1}(k, \bar{\ell})<0$ In this case, the bank chooses $\ell^{*}(k, \bar{\ell})=0$. For a symmetric equilibrium to exist in this case, we must have that $\bar{\ell}=0$, and for consistency, we must also have that $\alpha_{1}(k, 0)<0$. This situation corresponds to the case where the bank carries over no liquidity from time 0 .

Case 2: $\alpha_{1}(k, \bar{\ell})=0$ In this case, the bank is indifferent to the choice of $\ell$. For a symmetric equilibrium, the bank chooses $\ell^{*}(k, \bar{\ell})=\bar{\ell}$. The clearinghouse then implements

$$
\begin{gathered}
k^{*}=\underset{k}{\arg \max } \mathbb{E} \Pi\left(k, \ell^{*}(k)\right) \text { where } \\
\ell^{*}(k) \text { solves } \alpha_{1}\left(k, \ell^{*}(k)\right)=0
\end{gathered}
$$


Case 3: $\alpha_{1}(k, \bar{\ell})>0$ In this case, the bank chooses $\ell^{*}(k, \bar{\ell})=1$ and in equilibrium, $\ell^{*}=\bar{\ell}=1$. There is no systemic risk or investment in the risky asset and the clearinghouse never fails. This is an uninteresting case.

\section{Proposition 3.}

1. For every $\rho$ and for every $k$, there exists a unique $\ell^{*}(k)$ such that $\left(k, \ell^{*}(k)\right)$ is an equilibrium.

2. If $(k, \ell)$ is an equilibrium for a given $\rho$, then $(k+\ell, 0)$ is an equilibrium also.

3. For every $\rho, \ell^{*}\left(k^{*}\right)=0$, where $k^{*}=\arg \max _{k} \mathbb{E} \Pi\left(k, \ell^{*}(k)\right)$

Proof. See Appendix C.

The above results imply that at the optimal margin (for each value of $\rho$ ) chosen by the clearinghouse, banks do not carry over any liquidity. To better understand the gains from the clearinghouse and risk sharing, it is important to obtain the payoffs for each bank without the clearinghouse. In the absence of the insurance provided by the clearinghouse, banks may still choose to hold liquidity. Their liquidity holdings will allow them to purchase assets in a fire sale if they remain solvent, or may be large enough to help them refinance if they become insolvent, in which case a fire sale never takes place. In the proposition below, we show that the second situation always occurs, i.e., banks carry enough liquidity to refinance in the event of insolvency. Banks are therefore autarkic, meaning that they always refinance themselves if becoming insolvent.

Proposition 4. In the absence of the clearinghouse, banks choose liquidity levels $\ell^{*}=1 / 2$ for every $\rho$. There are no fire sales and banks have the same expected payoffs as under autarky. There is no systemic risk.

Proof. See Appendix D.

Our main result is that co-insurance is always strictly better than autarky in that it implies a higher expected profit for banks. However, for every possible equilibrium under coinsurance, there is higher systemic risk and greater investment in the risky asset than under autarky. Further, liquidity held by banks is sharply curtailed in the presence of a clearinghouse. This also means that if the clearinghouse fails, banks that do not get bailed out by the clearinghouse are worse off since other banks do not carry liquidity, and likely will have to sell their assets at a lower price.

The three panels of Figure 3 illustrate the equilibrium with the clearinghouse and fire sale for two separate values of $\tau$. The figure considers $R=1.5$, and $\alpha=0.4$. Under autarky, there is no systemic risk and payoffs are given by $(1+R-\alpha) / 2=1.05$.

The upper-left panel presents a plot of the initial margin as correlation varies. When correlation is very low, the optimal initial margin is such that the clearinghouse only fails with low probability. When $\tau$ is high, the margin is very low at low values of $\rho$ since the clearinghouse can pledge a large fraction of solvent banks' payoffs to refinance insolvent banks. On the other hand, when $\tau$ is low, it is optimal for banks to deposit a large margin into the clearinghouse to prevent failure. As correlation increases, more firms fail together, so reliance on successful investments alone is insufficient and the initial margin level becomes positive, eventually rising towards the autarky level of 0.50 . The investment in the risky asset under coinsurance is therefore equal to 1 , the maximum possible amount, at low levels of correlation but decreases towards the autarky level of $1 / 2$ as correlation increases. As $\tau$-the fraction of future payoffs that can be pledged-goes up, the optimal margin paid to the clearinghouse falls. As $\tau$ rises, it becomes more probable that the clearinghouse will not fail and, if it does, that the fire sale will happen at no arbitrage, i.e., with a price of $(R-\Delta-1)$, and insolvent banks will be better off. Banks internalize this and respond by depositing less in margin to the clearinghouse. 

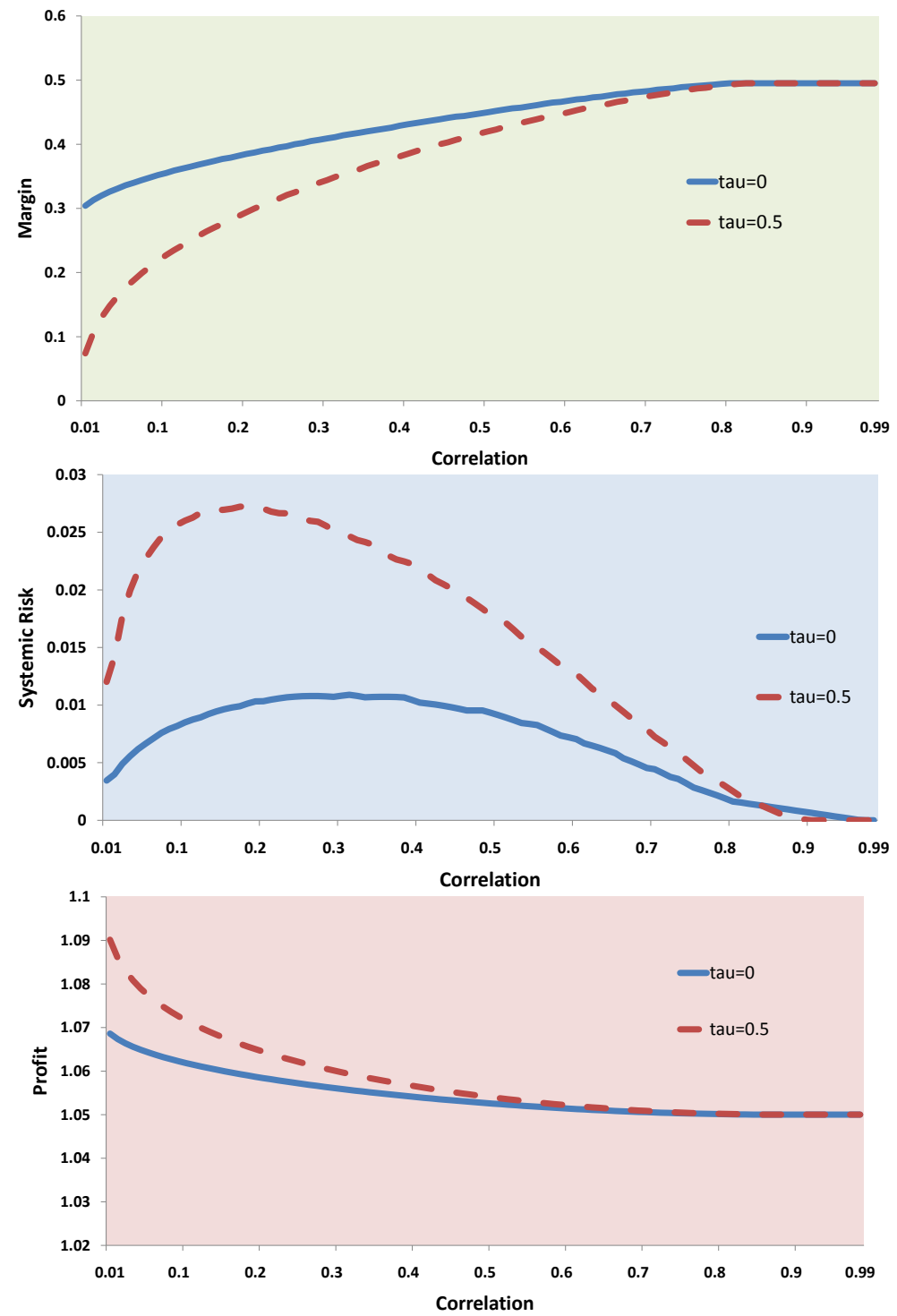

Figure 3. Equilibrium under co-insurance and fire sale. The three panels of this figure plot various properties of equilibrium by varying $\tau$ in the model of Section 5 . In all the figures, the return from the risky project is set to $R=1.50$ and the value of $\alpha$ (the probability that refinancing of the risky project will be required) is set to 0.4 . Panel 1 (top left) plots aggregate systemic liquidity in equilibrium as correlation changes. Panel 2 (top right) plots systemic risk as correlation changes. Lastly, Panel 3 (bottom) plots the payoff to a generic bank as correlation changes.

The upper right panel plots systemic risk in the model as a function of correlation. We define systemic risk as the ex-ante probability that a bank will fail and have to sell its assets inefficiently in a fire sale. For the initial low levels of correlation where the optimal initial margin is zero, this probability of failure increases with correlation. Beyond this point, the collection of initial margin causes the probability of failure to fall back down, eventually reaching the zero probability level of autarky. We see that systemic risk sharply rises as $\tau$ goes up. Since an increase in $\tau$ is accompanied by a lowering of the liquidity held by the clearinghouse, an inefficient fire sale becomes more likely leading to a rise in systemic risk. This is another illustration of the fact that as markets become more efficient, systemic risk may rise.

The lower panel presents a typical plot of payoffs in the clearinghouse model as correlation varies. The payoff with the clearinghouse is at least as large as that under autarky. As correlation increases, the benefits of risk-sharing decrease, so co-insurance payoffs fall towards the autarky payoff. 
Not surprisingly, the payoff rises with $\tau$ since the pledgeability of the clearinghouse and banks to acquire assets in the fire sale increases, and hence banks are strictly better off.

Recall that, for any given value of $\rho$, and any equilibrium pair $\left(k^{*}, \ell^{*}\right)$ chosen by banks, the thresholds $a_{0}(k, \ell), a_{1}(k, \ell)$ and $\underline{a}(k, \ell)$ determine whether the clearinghouse fails and-if so- the fire sale prices and payoffs for participating banks. We have been assuming that the aggregate shock $a$ follows a standard normal distribution, i.e., $a \sim \Phi(0,1)$. Therefore, we can obtain the 95th and 5th percentile realization of $a$, and obtain profits and systemic risk at these realizations using Equation (5), which shows the ex-ante expected payoffs for a bank according to the realization of the aggregate shock $a$, and whether there is a fire sale or not.

These are shown in the two panels of Figure 4. The dotted line in the first panel of the figure shows profits with a bad realization of aggregate shock $a$. For low values of $\rho$, equilibrium quantities $\left(k^{*}, \ell^{*}\right)$ are such that the clearinghouse does not fail for a 5th percentile realization of $a$. At $\rho$ of around 0.05 , this no longer holds, which is why we see a sudden jump downwards in profits and rise in systemic risk. The interpretations of profits and systemic risk with bad realizations of $a$ is similar to the interpretation of value at risk measures, i.e., they indicate how much fragility is present in the system if there is a tail event (in this case, a bad draw of $a$ ).
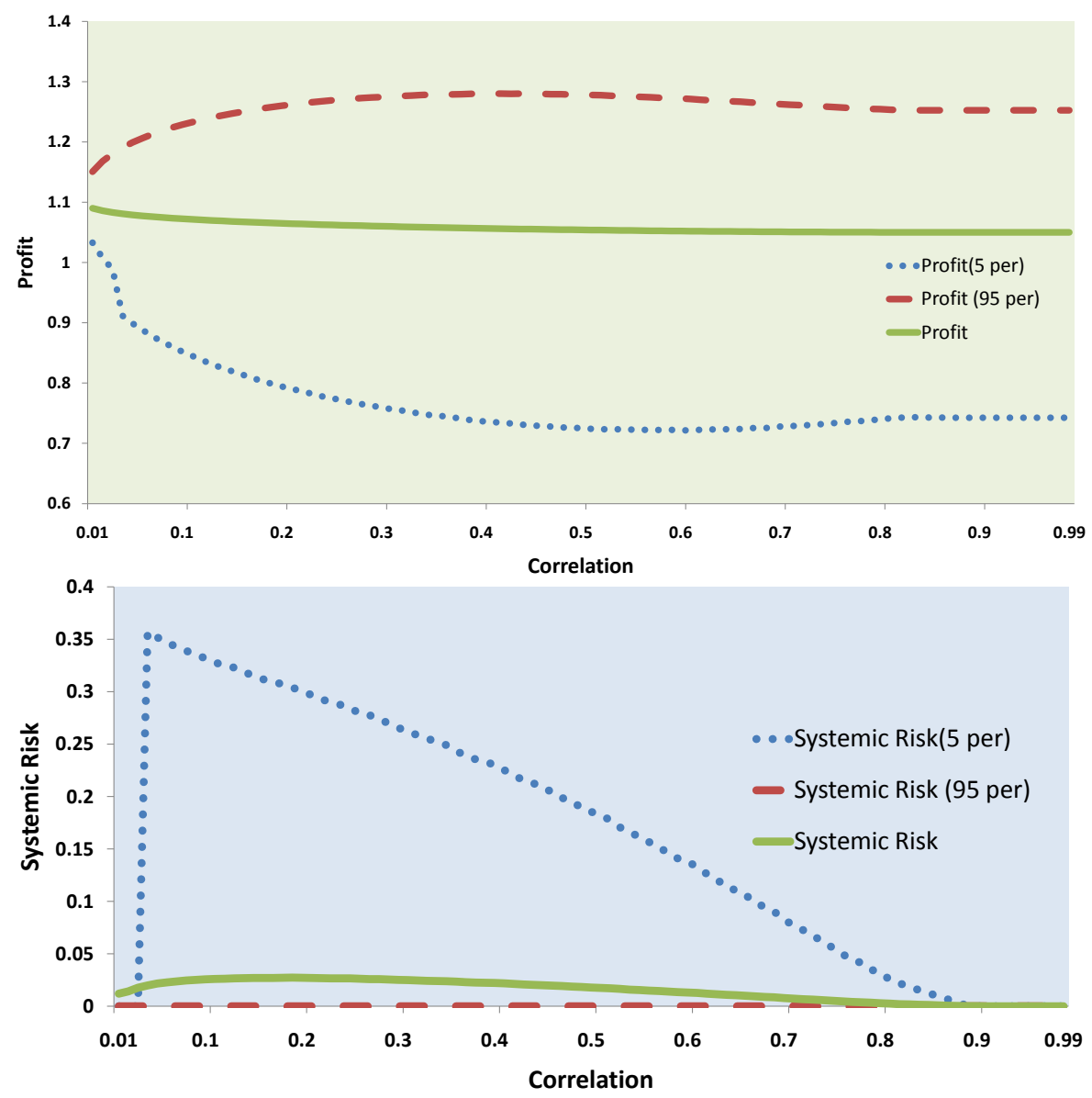

Figure 4. Dependence of profits and systemic risk on aggregate shock. The two panels of this figure indicate how profit and systemic risk is affected if "good" and "bad" aggregate shocks hit the economy. For a given value of $\rho$, "good" and "bad" shocks are defined as the 95th and 5th percentile realizations of the aggregate shock drawn from the distribution defined by $\rho$. As before, $R$ is set to 1.5 and $\alpha$ is set to 0.4. $\tau$ is set to 0.5. Panel 1 (top left) shows how profits change if a "good" or "bad" shock is realized, while Panel 2 (top right) plots the corresponding change in systemic risk. 


\subsection{Regulation and the First-Best Outcome}

The paper has so far assumed that banks take as given the liquidity choices of other banks and respond accordingly. In this section, we will consider the case where a regulator can mandate levels of margin and liquidity for each bank to carry. We will examine whether the solution to the regulators' problem differs from the equilibrium described in the previous section, and whether systemic risk is higher or lower as a result.

\section{Regulator's Problem:}

The regulator chooses margin $\left(k^{F B}\right)$ and liquidity $\left(\ell^{F B}\right)$ to maximize bank profits. In particular, $k^{F B}$ and $\ell^{F B}$ solve

$$
\left(k^{F B}, \ell^{F B}\right)=\underset{k, \ell}{\arg \max } \mathbb{E} \Pi^{F B}(k, \ell)
$$

where (as before $\underline{m}=\min \left(\underline{a}, a_{0}\right)$ and $\left.m_{1}=\min \left(a_{0}, a_{1}\right)\right)$

$$
\begin{aligned}
\mathbb{E}^{F B}(k, \ell)= & \\
& \int_{-\infty}^{a_{0}(k, \ell)}[R(1-k-\ell)+\ell](1-f(a)+g(k, \ell)) \phi(a) d a \\
& +\int_{-\infty}^{\underline{m}}[(R-\Delta-1) y(0, k, \ell)(1-f(a))+\ell(f(a)-g(k, \ell))] \phi(a) d a \\
& +\int_{\underline{m}}^{m_{1}}[(R-\Delta-1-p) y(p, k, \ell)(1-f(a))+[p \times(1-k-\ell)+\ell](f(a)-g(k, \ell))] \phi(a) d a \\
& +\int_{m_{1}}^{a_{0}(k, \ell)}[(R-\Delta-1)(1-k-\ell)+\ell)(f(a)-g(k, \ell)] \phi(a) d a \\
& +\int_{a_{0}(k, \ell)}^{\infty}([(\tau R(1-\eta(f(a)))+(1-\tau R))(1-f(a))+R f(a)](1-k-\ell)) \phi(a) d a
\end{aligned}
$$

The expression above for $\Pi^{F B}$ is similar to the expression for profit in the previous section. However, while earlier, banks chose liquidity $\ell$ taking as given the liquidity chosen by other banks $(\bar{\ell})$, here, the regulator solves for the optimal $\left(k^{F B}, \ell^{F B}\right)$ imposing as a condition that banks carry the same liquidity and contribute the same margin.

The question we are interested in is whether and to what extent regulatory requirements can improve welfare and lower systemic risk. This leads us to the following result:

\section{Proposition 5.}

1. For every value of correlation $\rho, k^{F B}(\rho)=k^{*}(\rho)$ and $\ell^{F B}(\rho)=\ell^{*}\left(k^{*}, \rho\right)=0$

2. For every value of $\rho, \mathbb{E} \Pi^{F B}(\rho)=\mathbb{E} \Pi(\rho)$, and systemic risk is as large under the first-best outcome as under equilibrium.

Proof. See Appendix E.

We see that the optimal values of margin and liquidity chosen by the regulator coincide with those obtained in equilibrium. Put another way, the equilibrium obtained in the previous section is ex-ante efficient and cannot be improved upon. In both the equilibrium and in the first-best case, liquidity carried over by banks equals zero. Regulators find it optimal to make banks deposit any non-risky investment as margin in the clearinghouse rather than carry as liquidity. The intuition for this result is that a dollar deposited in the clearinghouse as margin can be used to refinance banks without any loss in efficiency, i.e., assets refinanced by the clearinghouse generate a return of $R$. In contrast, liquidity carried over by banks helps to purchase assets in the fire sale, but this only generates a return of $(R-\Delta-1)$, i.e., there is some efficiency loss to acquiring new assets. It is therefore ex-ante optimal for regulators to mandate higher margins but no "carried-over" liquidity. 
As discussed earlier, it is also a property of equilibrium that at the optimal margin chosen by banks, liquidity carried over is zero. Thus, the first-best and equilibrium problems become identical if $\ell$ is zero, and optimal margins in both settings coincide. This in turn leads to identical profits and systemic risk in both cases.

\section{Model with Lender of Last Resort}

\subsection{Setting and Payoffs}

Let us assume that there is a government or a central bank that acts as a lender of last resort to the clearinghouse. There has been a precedent for a lender of last resort to bail out a clearinghouse. For example, after the 1987 stock market crash, the Federal Reserve extended credit to members of the Chicago Mercantile Exchange clearinghouse (CME).

Let the lender of last resort inject funds $g(a)$ into the clearinghouse, where $a$ is the realization of the aggregate shock. The cost to the government is given by $c(g)=a_{\text {gov }} g^{2}$. This cost can be thought of as arising from an increased tax levied by the government on the economy to fund the bailout.

If each bank submits margin $k$ and carries liquidity $\ell$, then each bank needs $(1-k-\ell)$ to refinance. The total number of banks that get refinanced by the government is $g(a) /(1-k-\ell)$.

Each bank that gets refinanced by the government sees a benefit of $\Delta$ per unit of asset refinanced ${ }^{9}$. Therefore, the total benefit to each bank from the government is $\Delta(1-k-\ell)$.

Since there are $g(a) /(1-k-\ell)$ banks that need refinancing, the total benefit to the economy through the government injecting liquidity is $\Delta g(a)$.

The cost function to the government is convex, and the government only injects funds when the marginal benefit is greater than the marginal cost, i.e.,

$$
g(a)<g^{*}, \text { where } c^{\prime}\left(g^{*}\right)=\Delta
$$

Let us assume that if the government acts as a lender of last resort, it still requires the clearinghouse to make capital calls on its members and refinance as many banks as possible. In the earlier version of the model, the clearinghouse could bail out a maximum of $f_{0}(k, \ell)$ banks, where

$$
f_{0}(k, \ell)=\frac{\tau R(1-k-\ell)+k+\ell}{(1+\tau R)(1-k-\ell)}
$$

The government can act as a lender of last resort to a maximum of $g^{*} /(1-k-\ell)$ banks.

Therefore, there is a fire sale only when the number of failures $f(a)>f_{g}=f_{0}+g^{*} /(1-k-\ell)$. In the case there is a fire sale, the clearinghouse uses margin $k$ to bail out $k /(1-k-\ell)$ banks, while the government acts as a lender of last resort for $g^{*} /(1-k-\ell)$ banks.

As before, if there is a fire sale, there are failure thresholds $\underline{f}$ and $f_{1}$ such that if $f>\underline{f}$, the fire sale price is zero, while if $f<f_{1}$, the price is $(R-\Delta-1)$. The thresholds are given by

$$
\underline{f}=\frac{\bar{\ell}+k+g^{*}+\tau R(1-k-\bar{\ell})}{(1-k-\bar{\ell})+(\bar{\ell}+\tau R(1-k-\bar{\ell}))} ; f_{1}=\frac{\bar{\ell}+\tau R(1-k-\bar{\ell})+(R-\Delta)\left(k+g^{*}\right)}{\bar{\ell}+\tau R(1-k-\bar{\ell})+(R-\Delta)(1-k-\bar{\ell})}
$$

Since the government acts as a lender of last resort, banks have to repay the government after getting refinanced. Therefore, if $f(a)>f_{g}$, there is a fire sale, the government injects $g^{*}$, and this is repaid to the government after some banks get refinanced.

If $f(a) \in\left(f_{0}, f_{g}\right]$, the government injects funds $g(a)=(1-k-\ell) \times\left(f(a)-f_{0}\right)$.

If $f(a)<f_{0}$, the clearinghouse remains solvent, and the government plays no role in the economy. 


\subsection{Expected Profits and Welfare}

Let $a_{0}, a_{1}, \underline{a}$ and $a_{g}$ be values of aggregate shock associated with failures $f_{0}, f_{1}, f$ and $f_{g}$ respectively. In addition, for notational ease, let $\underline{m}=\min \left(\underline{a}, a_{g}\right)$ and $m_{1}=\min \left(a_{g}, a_{1}\right)$.

Let $g_{1}\left(k, g^{*}, \bar{\ell}\right)=\left(k+g^{*}\right) /(1-k-\bar{\ell})$ be the number of banks either bailed out or refinanced by both the clearinghouse and the government if the clearinghouse fails.

Expected profits to banks in the presence of government acting as lender of last resort is given by

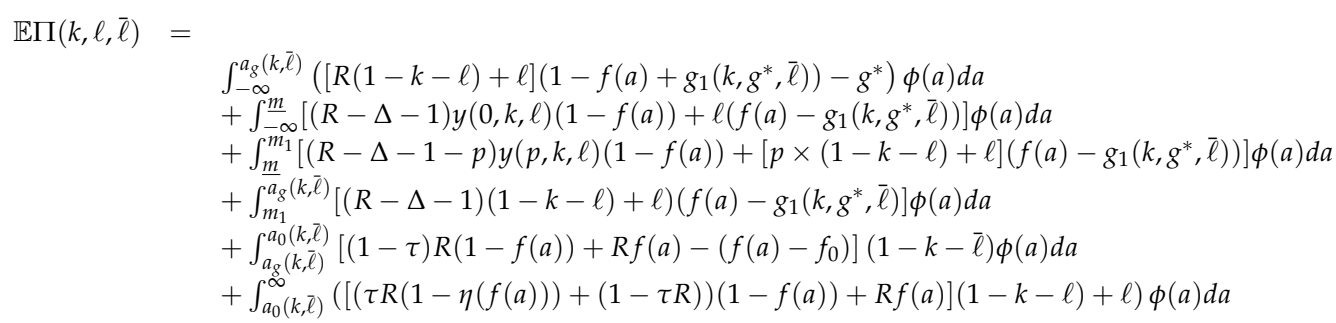

This expression is similar to our original expression for profit, except for the fact that more banks can get refinanced ( $g_{1}$ has changed), and banks have to repay the government after being refinanced.

The first term is the expected profit of banks from their own assets (without participating in the fire sale) if a fire sale takes place. For each realization of the aggregate shock $a,(1-f(a))$ banks remain solvent and do not need refinancing. $g_{1}$ insolvent banks do get refinanced using the margins present in the clearinghouse and injected government liquidity. Thus the number of banks that either do not need refinancing (and receive full return on their risky holdings) or do require refinancing and are refinanced is $\left(1-f(a)+g_{1}\left(k, g^{*}, \bar{\ell}\right)\right)$.

Finally, banks repay the government the injected amount $g^{*}$ after getting refinanced, giving the first term of the expression above.

The second, third and fourth terms capture expected payoffs from participating in the fire sale for both acquiring and selling banks. In the second term, the fire sale price is 0 , and so only acquiring banks realize a profit. In the third term, the fire sale price is some $p \in(0, R-\Delta-1)$. Both acquiring banks and banks that sell in the fire sale realize a payoff. Acquiring banks get $(R-\Delta-1-p)$ per unit of asset bought in the fire sale, while selling banks get price $p$ for the $(1-k-\ell)$ assets sold in the fire sale. The fourth term corresponds to profits when the price in the fire sale is $(R-\Delta-1)$. In this case, acquiring banks do not realize a profit in the fire sale.

The fifth term corresponds to expected profits when the clearinghouse has implemented maximum transfers $(\eta(f)=1)$ and the government still steps in to inject liquidity of $\left(f(a)-f_{0}\right)(1-k-\ell)$. Finally, the last term corresponds to the case where the clearinghouse remains solvent and there is no role for the government.

Welfare is given by

$$
W(k, \ell, \bar{\ell})=\mathbb{E} \Pi(k, \ell, \bar{\ell})-\Phi\left(a_{g}(k, \bar{\ell})\right) c\left(g^{*}\right)-\int_{a_{g}(k, \bar{\ell})}^{a_{0}(k, \bar{\ell})} a_{g o v}\left[f(a)-f_{0}\right]^{2}(1-k-\bar{\ell})^{2}
$$

where there is a welfare loss of $c\left(g^{*}\right)=a_{g o v}\left(g^{*}\right)^{2}$ if a fire sale happens (with probability $\left.\Phi\left(a_{g}(k, \bar{\ell})\right)\right)$ and the government uses $g^{*}$ to bail out banks, while there is a welfare loss of $a_{\text {gov }}\left[f(a)-f_{0}\right]^{2}(1-k-\bar{\ell})^{2}$ if $a \in\left[a_{0}, a_{g}\right]$, since in this region, the government has injected $g(a)=(1-k-\ell) \times\left(f(a)-f_{0}\right)$.

As before, $k_{p u b}$ is chosen to maximize welfare. First best liquidity will still always be zero.

Let $k_{p u b}$ be the margin mandated by the government to maximize welfare, and let $k_{p v t}$ be the optimal margin chosen by banks.

$$
k_{p u b}=\underset{k}{\arg \max } W(k, \bar{\ell}(k)) ; k_{p v t}=\underset{k}{\arg \max } \mathbb{E} \Pi(k, \bar{\ell}(k))
$$


The optimal margins chosen by the government and individual banks differ in that the government internalizes the costs of bank failures. This naturally makes the government more cautious than banks and we would expect the government to mandate higher margins than would be chosen privately. Systemic risk would correspondingly fall. This leads us to the following proposition:

\section{Proposition 6.}

$$
k_{p u b}>k_{\text {pvt }} ; \text { S.risk }_{p u b}<\text { S.risk } k_{p v t}
$$

Proof. See Appendix F.

The top left panel of Figure 5 shows how the margin changes with correlation for four separate cases-autarky, equilibrium in the absence of a government (acting as a lender of last resort), equilibrium in the presence of a government, and the first-best case.

The margin under autarky is fixed at 0.5. Banks opt to self-hedge and hold enough liquidity to refinance their project if the need arises. The rising solid line and (respt.) the dashed line plot optimal margins under the equilibrium without and (respt.) with the government. We note that margins are higher in the absence of the government. In the plots, the maximal amount of funds the government injects into the economy is $g^{*}=0.5$. This means that margins in the presence of the government never exceeds $\left(1 / 2-g^{*}\right)=0.45$. Banks know that they will be bailed out if needed and choose to correspondingly carry less in margin. The difference in margins carried with and without the government is highest when correlation is low. Margin under the first-best case is shown as the dotted line in the figure. This is always higher than margin under the private equilibrium in the presence of government, illustrating the proposition stated earlier.

The top right panel of the figure shows systemic risk for the four different cases discussed earlier. Systemic risk under autarky is just zero, while systemic risk with and without the government acting as lender of last resort are almost identical. While margins are lower with the government, the injection of public funds into the economy can lower the incidence and expected losses resulting from fire sales. Systemic risk is much lower, however, in the first-best outcome, which reflects the fact that margins are higher. The bottom panel shows how welfare varies with correlation for each of the four outcomes. Welfare under autarky is fixed at $(1+R-\alpha) / 2$, and, by definition, is highest under the first-best outcome. Further, welfare in the equilibrium with the government is higher than without.

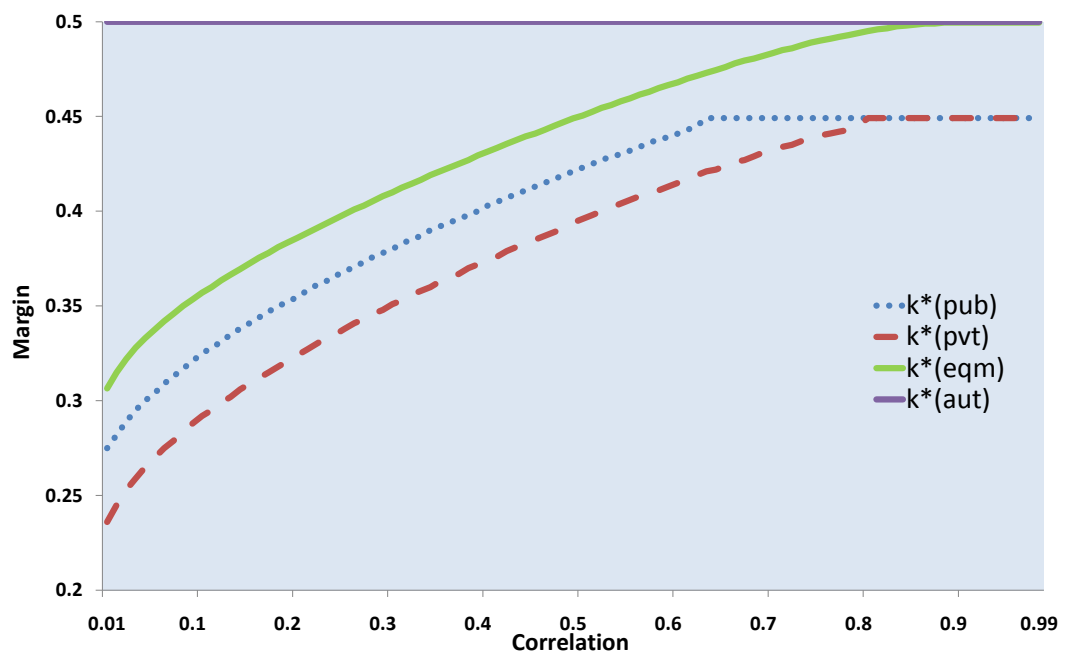

Figure 5. Cont. 

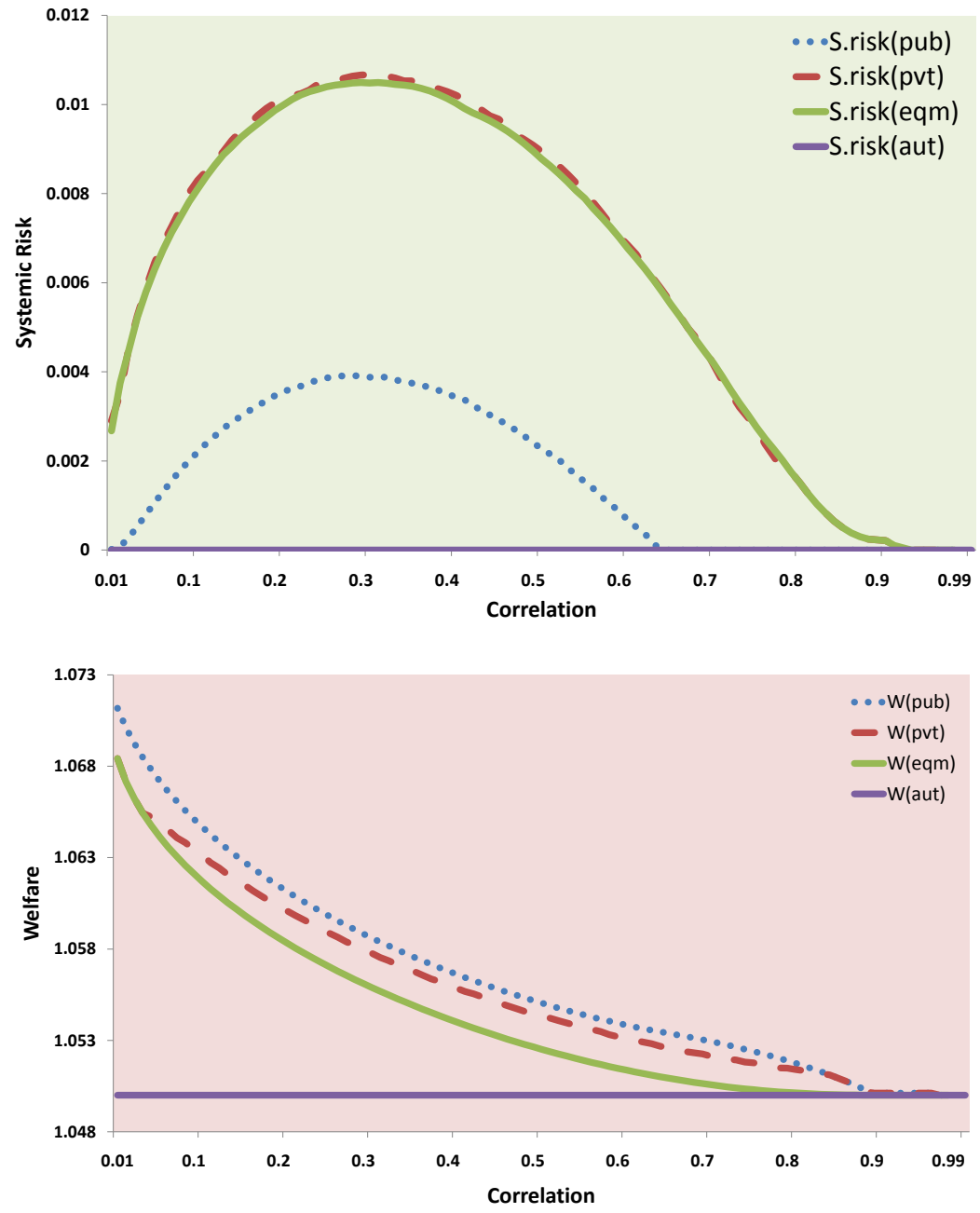

Figure 5. Outcomes with lender of last resort. The three panels of this figure plot outcomes under four different environments - autarky, equilibrium with and without the lender of last resort, and first-best. In all the figures, the return from the risky project is set to $R=1.50$ and the value of $\alpha$ (the probability that refinancing of the risky project will be required) is set to 0.4 . Panel 1 (top left) plots margin, $k$ as correlation changes. Panel 2 (top right) plots systemic risk, while Panel 3 (bottom) plots the payoff to a generic bank as correlation changes.

\section{Conclusions}

This paper makes the fundamental point that whether aggregate shocks lead to systemic risk or collective failure of financial firms depends on the liquidity choices of firms but that these liquidity choices are intimately shaped by the risk-sharing opportunities in the economy. When risk-sharing opportunities are abundant, holding liquidity to hedge against low-risk aggregate shocks is privately costly resulting in excessive investment and too little reserves in the system for dealing with such shocks. This novel insight helps resolve the paradox that risk-sharing appears to have led to greater systemic risk and incidence of financial crises. Interestingly, however, risk-sharing can lead to first-best outcomes and is efficient, even though it leads to increased systemic risk. In the presence of a lender of last resort, however, this is no longer true, and banks choose lower liquidity than is optimal under first-best. Important future work that remains is to understand how such regulation would affect the price of liquidity and the return on investments, both of which we took as given in this paper.

Author Contributions: Data curation, V.V.A. and A.M.I.; Formal analysis, R.K.S. All authors have read and agreed to the published version of the manuscript. 
Funding: This research received no external funding.

Acknowledgments: We are grateful to Stew Myers, participants at the Derivatives Research Project Day of the NYU Stern School of Business, participants at the "Challenges in Interconnected Financial Systems" at the Sveriges Riksbank, the Systemic Risk Center at LSE, and the Commodities Futures Trading Commission for their feedback.

Conflicts of Interest: The authors declare no conflict of interest.

\section{Appendix A. Proof of Proposition 1}

Pick a strategy $(\ell, 1-\ell)$. Since refinancing costs are a dollar per dollar of investment in the risky asset, the total refinancing need (should a refinancing need arise) will be $(1-\ell)$. Since only internal resources may be used to refinance, this means the bank can refinance the risky investment if and only if $\ell \geq(1-\ell)$, i.e., if and only if $\ell \geq 1 / 2$.

If the bank chooses $\ell<1 / 2$, then refinancing is impossible, and investment in the risky project yields a return of $R$ (per dollar investment) with probability $1-\alpha$ and 0 with probability $\alpha$. Since the riskless project has a return of 1 (per dollar investment) with certainty, the expected payoff from the strategy $(\ell, 1-\ell)$ is

$$
\ell+(1-\alpha)(1-\ell) R=\ell[1-R+\alpha R]+(1-\alpha) R .
$$

On the other hand, if the bank chooses $\ell \geq 1 / 2$, then refinancing is always feasible. Since refinancing is optimal when it is feasible, and since refinancing costs of a dollar (per dollar investment) are incurred with probability $\alpha$, the expected payoff per dollar of investment in the risky strategy is $R-\alpha$. So the payoff from the strategy $(\ell, 1-\ell)$, conditional on choosing $\ell \geq 1 / 2$, is

$$
\ell+(1-\ell) R-\alpha(1-\ell)=\ell[1-R+\alpha]+R-\alpha .
$$

To identify the optimal strategy for the bank, we compare the maximized values of the payoffs (A1) and (A2), i.e., (i) the maximal payoff conditional on $\ell<1 / 2$, and (ii) the maximal payoff conditional on $\ell \geq 1 / 2$. Consider expression (A1) first. It is apparent from visual inspection that the optimal action is to choose $\ell^{*}=0$ if $1-R+\alpha R \leq 0$, and to choose $\ell^{*}$ as high as possible (subject to $\ell<1 / 2$ ) if $1-R+\alpha R>0$. Thus, the maximum value of the payoff (or the supremum since there is no maximum in the latter case) is

$$
\begin{cases}(1-\alpha) R, & \text { if } 1-R+\alpha R \leq 0 \\ \frac{1}{2}[1+R-\alpha R], & \text { if } 1-R+\alpha R>0\end{cases}
$$

Now consider the choice of $\ell$ that maximizes expression (A2) subject to $\ell \geq 1 / 2$. Again, simple visual inspection shows that the optimal action is the smallest possible value of $\ell$ (which is $\ell^{*}=1 / 2$ ) if $1-R+\alpha \leq 0$ and the largest possible value of $\ell$ (which is $\ell^{*}=1$ ) if $1-R+\alpha \geq 0$. Thus, the maximized value of the payoff is

$$
\begin{cases}\frac{1}{2}[1+R-\alpha], & \text { if } 1-R+\alpha \leq 0 \\ 1, & \text { if } 1-R+\alpha>0\end{cases}
$$

Since $R>1$, this gives this three cases to consider:

- Case 1. $0 \geq 1-R+\alpha R>1-R+\alpha$. 
In this case, the maximized payoff under $\ell<1 / 2$ is $(1-\alpha) R$, which corresponds to a choice of $\ell^{*}=0$, while the maximized payoff under $\ell \geq 1 / 2$ is

$$
\frac{1}{2}[1+R-\alpha]
$$

The former is greater if and only if $2(1-\alpha) R>1+R-\alpha$, i.e., if and only if

$$
\alpha<\frac{R-1}{2 R-1}=\lambda(R)
$$

Thus, for $\alpha<\lambda(R)$, the optimal action is $\ell^{*}=0$ leading to a payoff of $\left.1-\alpha\right) R$ if $\alpha<\lambda(R)$, while the optimal action is $\ell^{*}=1 / 2$ leading to a payoff of (A3) if $\alpha \in[\lambda(R),(R-1) / R]$. This establishes Part 1 of the proposition.

- Case 2. $1-R+\alpha R>0>1-R+\alpha$.

Note that $1-R+\alpha<0 \Longleftrightarrow \alpha<v(R)$ as defined in the statement of Proposition 1 . The supremum of the payoffs (there is no maximum) subject to $\ell<1 / 2$ here is

$$
\frac{1}{2}[1+R-\alpha R]
$$

while the maximized value of the payoffs subject to $\ell \geq 1 / 2$ (corresponding to the action $\ell^{*}=1 / 2$ ) is the same as given by (A3). Since $R>1$, the latter is always greater, so it remains optimal to choose $\ell^{*}=1 / 2$ and thence to obtain the expected payoff (A3). This establishes Part 2 of the proposition.

- Case 3. $1-R+\alpha R>1-R+\alpha \geq 0$.

In this case, the supremum of the payoffs (there is no maximum) subject to $\ell<1 / 2$ continues to be given by (A4), while the maximized payoff subject to $\ell \geq 1 / 2$, corresponding to the optimal action $\ell^{*}=1$, is 1 . The latter is greater as long as

$$
1+R-\alpha R<2 \Longleftrightarrow R(1-\alpha)<1
$$

However, this always holds since we are considering the case $1-R+\alpha R>0$. Thus, the optimal action in this case is to set $\ell^{*}=1$ and to receive an expected payoff of 1 . This establishes Part 3 and completes the proof of the proposition.

\section{Appendix B. Proof of Proposition 2}

Recall that when $\alpha \in[\lambda(R), v(R)]$ (the case we are considering), under autarky each bank invests an amount of $1 / 2$ in the riskless asset and $1 / 2$ in the risky project, and receives an ex-ante expected payoff of

$$
\frac{1}{2}(1+R-\alpha)
$$

Consider the following strategies for the two banks that corresponds to a "mutualization" of risk (equivalently, to each bank buying a specific contingent claim from the other bank):

1. Each bank contributes an amount $\ell^{*}=1 / 3$ to a common insurance "pool." The pool is invested in the riskless asset.

2. Each bank invests its remaining funds $1-\ell^{*}=2 / 3$ in its risky project.

3. Either bank can claim the amount in the pool if its risky project requires refinancing. (Recall that the refinancing need is observable and verifiable, so false claims cannot be made.)

4. If more than one bank makes a claim at the same time, then the banks share the available money equally. 
5. If neither bank experiences a refinancing need, the available money in the pool is equally divided between the two banks at time 2 .

Since each bank invests $2 / 3$ in the risky project, and there is a total of $2 / 3$ invested in the riskless asset, there is exactly enough to refinance one project but not both. So if only one bank needs refinancing (which happens in States 2 and 3), the project can be refinanced, but if both banks need refinancing (State 4), refinancing is impossible and there is "systemic" failure. Note too that if either bank deviates unilaterally to a lower contribution to the pool, there will not be enough cash to refinance even one project, so the only possible deviation that could dominate the chosen allocation is autarky, a point we will return to shortly.

The state-wise payoff from the specified strategies for each bank is:

\begin{tabular}{cc}
\hline State & Payoff \\
\hline 1 & $1 / 3+2 R / 3$ \\
2 & $2 R / 3$ \\
3 & $2 R / 3$ \\
4 & $1 / 3$ \\
\hline
\end{tabular}

So the expected payoff to each bank from the described strategies is

$$
\frac{1}{3}\left(p_{1}+p_{4}\right)+\frac{2}{3}\left(1-p_{4}\right) R,
$$

or, substituting for the probabilities $p_{i}$,

$$
\frac{1}{3}[1-2(1-\rho) \alpha(1-\alpha)]+\frac{2}{3}(1-\alpha)(1+\alpha-\alpha \rho) R
$$

This risk-sharing payoff (A7) dominates the autarky payoff (A5) as long as

$$
\left\{\frac{1}{3}[1-2(1-\rho) \alpha(1-\alpha)]+\frac{2}{3}(1-\alpha)(1+\alpha-\alpha \rho) R\right\} \geq \frac{1}{2}(1+R-\alpha)
$$

A little bit of algebra shows that this condition is equivalent to the condition that

$$
(R-1)[1-4 \alpha(\alpha+\rho-\alpha \rho)] \geq \alpha
$$

When does (A8) hold? As $\rho \downarrow 0$, this reduces to the condition that

$$
(R-1)\left(1-4 \alpha^{2}\right) \geq \alpha
$$

Now, as $\alpha$ approaches its lower bound $\lambda(R)=(R-1) /(2 R-1)$, the left-hand side of (A9) tends to

$$
\left(\frac{R-1}{2 R-1}\right)\left(\frac{4 R-3}{2 R-1}\right)
$$

while the right-hand side approaches $(R-1) /(2 R-1)$. Therefore, in the limit, the left-hand side is strictly larger as long as

$$
\frac{4 R-3}{2 R-1}>1,
$$

which always holds since $R>1$. Since the inequality holds strictly, it follows that there exists an interval $\left[\lambda(R), \alpha^{*}\right)$ such that for $\alpha$ in this interval, risk-sharing strictly dominates the autarky payoff.

It is easy to see from this that for any such $\alpha$ the proposed strategies constitute an equilibrium. From the dominance, neither party will prefer to revert to an autarkic equilibrium. Neither party will also wish to deviate to a lower (but positive) contribution to the pool because there will then not be 
enough resources in the pool to refinance even one project. Neither party will wish to deviate to a zero contribution since payoff from a zero contribution leads, at best, to the autarkic payoff which is dominated by the proposed risk-sharing strategies.

By continuity in $\rho$, it follows that for all suitably small $\rho$, there is an interval $\left[\lambda(R), \alpha^{*}\right)$ of possible values of $\alpha$ such that the risk-sharing equilibrium dominates autarky for all $\alpha$ in this interval. The upper-bound $\alpha^{*}$ depends on $\rho$ (and indeed, declines in $\rho$, since it is easily checked that the payoff (A7) from risk-sharing is declining in $\rho$ ). ${ }^{10}$

At high correlations, however, the situation reverses itself, i.e., autarky dominates the payoff under risk-sharing:

$$
\frac{1}{3}[1-2(1-\rho) \alpha(1-\alpha)]+\frac{2}{3}(1-\alpha)(1+\alpha-\alpha \rho) R<\frac{1}{2}(1+R-\alpha) .
$$

To see (A10), note that as $\rho \uparrow 1$, the left-hand side tends to the limit

$$
\frac{1}{3}+\frac{2}{3}(1-\alpha) R
$$

and this is strictly less than the right-hand side of (A10) if

$$
2+4(1-\alpha) R<3(1+R-\alpha)
$$

i.e., if $\alpha>(R-1) /(4 R-3)$. However, $\alpha>(R-1) /(2 R-1)$ by hypothesis, and $(2 R-1)<(4 R-3)$, so certainly $\alpha>(R-1) /(4 R-3)$, so the strict inequality (A10) holds in the limit. Once again, by continuity in $\rho$, it follows that for sufficiently high $\rho$, autarky dominates risk-sharing.

Finally, as observed earlier, the expected payoff from risk-sharing (A7) is strictly decreasing in $\rho$ : differentiating this payoff with respect to $\rho$, we obtain

$$
\frac{2}{3} \alpha(1-\alpha)-\frac{2}{3} \alpha(1-\alpha) R=\frac{2}{3} \alpha(1-\alpha)(1-R)<0
$$

It follows that there is a unique $\rho^{*}$ such that (a) for $\rho>\rho^{*}$, autarky dominates risk-sharing for all $\alpha \geq \lambda(R)$, while (b) for $\rho<\rho^{*}$, there is an interval $\left[\lambda(R), \alpha^{*}\right)$ of possible values of $\alpha$ such that risk-sharing dominates autarky for all $\alpha$ in this interval. Since $\alpha^{*}$ declines in $\rho$, we can solve explicitly for $\rho^{*}$ by taking $\alpha$ equal to $\lambda(R)$ and setting the two sides of (A8) equal:

$$
(R-1)\left[1-4 \frac{(R-1)^{2}}{(2 R-1)^{2}}-4 \rho^{*} \frac{R-1}{2 R-1}\left(1-\frac{R-1}{2 R-1}\right)\right]=\frac{R-1}{2 R-1}
$$

or, rearranging and canceling common terms,

$$
\left[1-4 \frac{(R-1)^{2}}{(2 R-1)^{2}}-4 \rho^{*} \frac{R(R-1)}{(2 R-1)^{2}}\right]=\frac{1}{2 R-1}
$$

Multiplying through by $2 R-1$,

$$
2 R-1-4 \frac{(R-1)^{2}}{2 R-1}-4 \rho^{*} \frac{R(R-1)}{2 R-1}=1
$$

So

$$
2 R-2=4 \frac{(R-1)^{2}}{2 R-1}+4 \rho^{*} \frac{R(R-1)}{2 R-1}
$$

10 How large can $\alpha^{*}$ be? It must clearly be strictly less than $1 / 2$, since (A9) clearly fails for $\alpha \geq 1 / 2$. 
Multiplying through again by $(2 R-1)$ and re-arranging, we have

$$
2(R-1)(2 R-1)-4(R-1)^{2}=4 \rho^{*} R(R-1)
$$

The left-hand side of this expression is $2(R-1)[(2 R-1)-2(R-1)]=2(R-1)$, so we obtain $2(R-1)=4 \rho^{*} R(R-1)$, which finally gives us

$$
\rho^{*}=\frac{1}{2 R}
$$

This completes the proof of Proposition 2.

Note on Choice of $\varphi$ :

A natural question to ask is whether the results in the above proposition continue to hold if $\varphi<1$. It turns out that they do, and that there continues to exist an equilibrium in which risk sharing can lead to greater systemic risk. In particular, $\lambda(R, \varphi)$ and $v(R, \varphi)$ in the 1-bank model become $\varphi(R-1) /(R+\varphi(R-1))$ and $(R-1) / \varphi$, respectively.

In the 2-bank model, the optimal choice of liquidity carried in the case of risk-sharing, $\ell^{*}=$ $\varphi /(2+\varphi)$. With these expressions in place, we can verify that the results from the previous proposition, namely:

1. For small enough $\rho$ there always exists an $\alpha^{*}$ such that for $\alpha \in\left[\lambda(R, \varphi), \alpha^{*}\right]$, risk sharing dominates autarky, and

2. $\exists \rho^{*}$ such that for $\rho<\rho^{*}$, there is an equilibrium where risk-sharing dominates autarky continue to hold.

\section{Appendix C. Proof of Proposition 3}

Let us define

$$
\begin{gathered}
T_{1}(a, k, \bar{\ell})=-R(1-f(a)+g(k, \bar{\ell})) \\
T_{2}(a, k, \bar{\ell})=(R-\Delta-1)(1-\tau R)(1-f(a)) \\
T_{3}(a, k, \bar{\ell})=\frac{(R-\Delta)(1-k-\bar{\ell})[f(a)-g(k, \bar{\ell})](1-\tau R)}{[\bar{\ell}+\tau R(1-k-\bar{\ell})]}-\frac{(1-k)}{(1-k-\bar{\ell})}(1-f(a))+f(a)-g(k, \bar{\ell}) \\
T_{4}(a, k, \bar{\ell})=-(R-\Delta-1)(f(a)-g(k, \bar{\ell})) \\
T_{\text {solv }}(a, k, \bar{\ell})=-\left[R-f(a)+\frac{k+\bar{\ell}}{1-k-\bar{\ell}}\right]
\end{gathered}
$$

Then,

$$
\begin{aligned}
\alpha_{1}(k, \bar{\ell}) & =\int_{-\infty}^{a_{0}(k, \bar{\ell})} T_{1}(a, k, \bar{\ell}) \phi(a) d a+\int_{-\infty}^{\underline{m}} T_{2}(a, k, \bar{\ell}) \phi(a) d a+\int_{\underline{m}}^{m_{1}} T_{3}(a, k, \bar{\ell}) \phi(a) d a \\
& +\int_{m_{1}}^{a_{0}(k, \bar{\ell})} T_{4}(a, k, \bar{\ell}) \phi(a) d a+\int_{a_{0}(k, \bar{\ell})}^{\infty} T_{\text {solv }}(a, k, \bar{\ell}) \phi(a) d a+1
\end{aligned}
$$

The expressions for $T_{1}, T_{2}, T_{4}$ and $T_{\text {solv }}$ are immediate. To see why the expression for $T_{3}$ must be true, note that $T_{3}(k, \bar{\ell})$ is the coefficient of $\ell$ in the expression $[(R-\Delta-1-p) y(p, k, \ell)(1-f(a))+[p \times$ $(1-k-\ell)+\ell](f(a)-g(k, \ell))]$ Now,

$$
\begin{gathered}
y(p, k, \ell)=\frac{\ell+\tau R(1-k-\ell)}{(1+p)} \text {, and } \\
p(k, \bar{\ell})=\max \left(0,-1+[\bar{\ell}+\tau R(1-k-\bar{\ell})] \frac{(1-f(a))}{(1-k-\bar{\ell})(f(a)-g(k, \bar{\ell}))}\right)
\end{gathered}
$$


This means that the coefficient of $\ell$ in $(R-\Delta) y(p, k, \ell)(1-f(a))$ is $(R-\Delta)(1-f(a))(1-\tau R) /(1+p)$, which in turn equals $(R-\Delta)(1-k-\bar{\ell})[f(a)-g(k, \bar{\ell})](1-\tau R) /[\bar{\ell}+\tau R(1-k-\bar{\ell})]$

Furthermore, the coefficient of $\ell$ in $(1+p) y(p, k, \ell)(1-f(a))+p(k, \bar{\ell})(1-k-\ell)(f(a)-g(k, \bar{\ell}))$ equals $(1-\tau R)(1-f(a))+p(k, \bar{\ell})(f(a)-g(k, \bar{\ell}))$.

After doing some algebra, and substituting the value of $p(k, \bar{\ell})$, we find that this reduces to

$$
(1-\tau R)(1-f(a))+p(k, \bar{\ell})(f(a)-g(k, \bar{\ell}))=(1-f(a)) \frac{(1-k)}{(1-k-\bar{\ell})}+f(a)-g(k, \bar{\ell})
$$

Now we are in a position to derive comparative statics on $\alpha_{1}(k, \bar{\ell})$.

Claim: $\partial \alpha_{1}(k, \bar{\ell}) / \partial \bar{\ell}<0$

Proof. First note that $\partial g(k, \bar{\ell}) / \partial \bar{\ell}>0$. This is immediate from the definition of $g(k, \bar{\ell})=k /(1-k-\bar{\ell})$.

This means that $\partial T_{1}(a, k, \bar{\ell}) / \partial \bar{\ell}<0$ and that $\partial T_{4}(a, k, \bar{\ell}) / \partial \bar{\ell}<0$. Further, it is clear that $\partial T_{\text {solv }}(a, k, \bar{\ell}) / \partial \bar{\ell}<0$

We will show that $\partial T_{3}(a, k, \bar{\ell}) / \partial \bar{\ell}<0$. First, note that

$$
\begin{aligned}
& \frac{d}{d \bar{\ell}} \frac{(R-\Delta)(1-k-\bar{\ell})[f(a)-g(k, \bar{\ell})](1-\tau R)}{[\bar{\ell}+\tau R(1-k-\bar{\ell})]} \\
& =\frac{d}{d \bar{\ell}}\left[\frac{f(a)-g(k, \bar{\ell})}{\bar{\ell} /(1-k-\bar{\ell})+\tau R}\right]=\frac{d}{d \bar{\ell}}\left[\frac{(1-k-\bar{\ell}) f(a)-k}{\bar{\ell}+\tau R(1-k-\bar{\ell})}\right] \\
& =\frac{-[\bar{\ell}+\tau R(1-k-\bar{\ell})] f(a)-[(1-k-\bar{\ell}) f(a)-k](1-\tau R)}{(\bar{\ell}+\tau R(1-k-\bar{\ell}))^{2}} \\
& =\frac{k(1-\tau R)-(1-k) f(a)}{(\bar{\ell}+\tau R(1-k-\bar{\ell}))^{2}}
\end{aligned}
$$

Now, for $a \in\left[\underline{m}, \underline{m_{1}}\right]$, we have that $f(a)>f_{1}(k, \bar{\ell})$. Therefore,

$$
f(a)>\frac{(\bar{\ell}+\tau R(1-k-\bar{\ell}))+k(R-\Delta)}{\bar{\ell}+\tau R(1-k-\bar{\ell})+(1-k-\bar{\ell})(R-\Delta)}>\frac{k}{(1-k-\bar{\ell})}
$$

This implies that $k(1-\tau R)-(1-k) f(a)<0$, and so

$$
\frac{d}{d \bar{\ell}} \frac{(R-\Delta)(1-k-\bar{\ell})[f(a)-g(k, \bar{\ell})](1-\tau R)}{[\bar{\ell}+\tau R(1-k-\bar{\ell})]}<0
$$

Therefore,

$$
\frac{\partial T_{3}(a, k, \bar{\ell})}{\partial \bar{\ell}}=\frac{d}{d \bar{\ell}} \frac{(R-\Delta)(1-k-\bar{\ell})[f(a)-g(k, \bar{\ell})](1-\tau R)}{[\bar{\ell}+\tau R(1-k-\bar{\ell})]}-\frac{d}{d \bar{\ell}} \frac{(1-k)(1-f(a))+k)}{(1-k-\bar{\ell})}<0
$$

We have now shown that $\partial T_{i} / \partial \bar{\ell}<0$ for $1 \leq i \leq 4$. We also know that $\partial T_{\text {solv }} / \partial \bar{\ell}<0$.

In our representation earlier, $\alpha_{1}(k, \bar{\ell})$ has been written as the sum of a series of definite integrals. By the Leibniz rule, $\partial \alpha_{1}(k, \bar{\ell}) / \partial \bar{\ell}=p_{1}(k, \bar{\ell})+p_{2}(k, \bar{\ell})$ where $p_{1}(k, \bar{\ell})$ is the sum of the integrals of the derivatives of the integrands, while $p_{2}(k, \bar{\ell})$ is the derivative of the integral limit, multiplied by the value of the integrand at this limit.

Therefore, in this case,

$$
p_{2}(k, \bar{\ell})=\frac{\partial a_{0}}{\partial \ell}\left[T_{1}\left(a_{0}, k, \bar{\ell}\right)+T_{2}\left(a_{0}, k, \bar{\ell}\right) \mathbb{1}_{\underline{m}=a_{0}}+T_{3}\left(a_{0}, k, \bar{\ell}\right) \mathbb{1}_{\underline{m}<a_{0} a n d m_{1}=a_{0}}+T_{4}\left(a_{0}, k, \bar{\ell}\right) \mathbb{1}_{\underline{m}, m_{1}<a_{0}}-T_{\text {solv }}\left(a_{0}, k, \bar{\ell}\right)\right]
$$


Now

$$
\begin{aligned}
T_{1}(a, k, \bar{\ell})+T_{4}(a, k, \bar{\ell})-T_{\text {solv }}(a, k, \bar{\ell}) & =-R(1-f(a)+g(k, \bar{\ell})-(R-1-\Delta)(f(a)-g(k, \bar{\ell})) \\
& +R-f(a)+g(k, \bar{\ell}) \\
& =\Delta(f(a)-g(k, \bar{\ell})) \\
& >0
\end{aligned}
$$

In addition, recall that $T_{3}(a, k, \bar{\ell})$ can be written as

$$
\begin{aligned}
T_{3}(a, k, \bar{\ell}) & =\frac{(R-\Delta)(1-f(a))(1-\tau R)}{(1+p)}-(1-\tau R)(1-f(a))-p(k, \bar{\ell})(f(a)-g(k, \bar{\ell})) \\
& >-p(k, \bar{\ell})(f(a)-g(k, \bar{\ell}) \\
& >-(R-\Delta-1)(f(a)-g(k, \bar{\ell}) \\
& =T_{4}(a, k, \bar{\ell})
\end{aligned}
$$

where both inequalities follow from the fact that fire sale price $p(k, \bar{\ell})$ is always less than or equal to $(R-\Delta-1)$.

By the same token, we can write

$$
\begin{aligned}
T_{3}(a, k, \bar{\ell}) & =\frac{(R-\Delta)(1-f(a))(1-\tau R)}{(1+p)}-(1-\tau R)(1-f(a))-p(k, \bar{\ell})(f(a)-g(k, \bar{\ell})) \\
& <(R-\Delta)(1-f(a))(1-\tau R)-(1-\tau R)(1-f(a)) \\
& =(R-\Delta-1)(1-f(a))(1-\tau R) \\
& =T_{2}(a, k, \bar{\ell})
\end{aligned}
$$

where the first inequality follows since price $p(k, \bar{\ell}) \geq 0$

Putting it all together, we have that $T_{4}(a, k, \bar{\ell})<T_{3}(a, k, \bar{\ell})<T_{2}(a, k, \bar{\ell}$, and so

$$
T_{1}(a, k, \bar{\ell})+T_{2}(a, k, \bar{\ell})-T_{\text {solv }}(a, k, \bar{\ell}) \geq 0
$$

and

$$
T_{1}(a, k, \bar{\ell})+T_{3}(a, k, \bar{\ell})-T_{\text {solv }}(a, k, \bar{\ell}) \geq 0
$$

Finally, $\partial a_{0}(k, \bar{\ell}) / \partial \bar{\ell}<0$ implies that $p_{2}(k, \bar{\ell})<0$. We have already shown that $p_{1}(k, \bar{\ell})<0$, and so

$$
\frac{\partial \alpha_{1}(k, \bar{\ell})}{\partial \bar{\ell}}<0,
$$

This shows that $\alpha_{1}(k, \bar{\ell})$ is a decreasing function. To prove uniqueness of equilibrium, all we have to do is show that there exists some limit $\tilde{\ell}$ at which $\alpha_{1}(k, \tilde{\ell}<0$. This would mean that either there exists a unique $\ell^{*} \in(0, \tilde{\ell})$ for which $\alpha_{1}\left(k, \ell^{*}\right)=0$, or it would mean that $\alpha_{1}(k, x)<0$ for all $x<\tilde{\ell}$, in which case $\ell^{*}=0$ is an equilibrium, as discussed earlier.

Let us choose $\tilde{\ell}$ such that $k /(1-k-\tilde{\ell})=1$. Then, the clearinghouse never fails. $a_{0}(k, \tilde{\ell})=-\infty$, and it is easy to see that $\alpha_{1}(k, \tilde{\ell})<0$.

Proof of part b. Here, we will show that if $(k, \bar{\ell})$ is an equilibrium, then $(k+\bar{\ell}, 0)$ is an equilibrium also. To prove this, we will show that $\mathcal{L}\left(\alpha_{1}(k, \bar{\ell})<0\right.$, where $\mathcal{L}(f(a, b))=\partial f / \partial a-\partial f / \partial b$. Note that proving this fact will imply the result since if $(k, \bar{\ell})$ is an equilibrium (and $\bar{\ell}>0$ ), it means that $\alpha_{1}(k, \bar{\ell})=0$, and the result would imply that $\alpha_{1}(k+\bar{\ell}, 0)<0$, which would imply $(k+\bar{\ell}, 0)$ is an equilibrium.

The outline of the proof is similar to before. First, we will prove that $\mathcal{L}\left(T_{i}\right)<0$ for $1 \leq i \leq 4$, and also that $\mathcal{L}\left(T_{\text {solv }}\right)<0$. We will then use Leibniz' rule to complete the proof. 
Note that $\mathcal{L}(f(k, \bar{\ell}))=\partial f / \partial k-\partial f / \partial \bar{\ell}=\partial f /\left.\partial k\right|_{k+\bar{\ell}=c}$ where $c$ is a constant. This interpretation will help us in some of the steps to follow.

It is clear that $\mathcal{L}(g(k, \bar{\ell}))=\mathcal{L}(k /(1-k-\bar{\ell}))>0$. This immediately implies that

$$
\mathcal{L}\left(T_{1}\right)<0 ; \mathcal{L}\left(T_{4}\right)<0 ; \mathcal{L}\left(T_{\text {solv }}\right)<0
$$

Since $\mathcal{L}\left(T_{2}\right)=0$, it only remains to prove that $\mathcal{L}\left(T_{3}\right)<0$.

Recall that

$$
T_{3}(a, k, \bar{\ell})=\frac{(R-\Delta)(1-k-\bar{\ell})[f(a)-g(k, \bar{\ell})](1-\tau R)}{[\bar{\ell}+\tau R(1-k-\bar{\ell})]}-\frac{(1-k)}{(1-k-\bar{\ell})}(1-f(a))+f(a)-g(k, \bar{\ell})
$$

We have that

$$
\mathcal{L}\left(-\frac{(1-k)}{(1-k-\bar{\ell})}(1-f(a))+f(a)-g(k, \bar{\ell})\right)=-\mathcal{L}\left(\frac{(1-k)(1-f(a))+k}{1-k-\bar{\ell}}\right)<0
$$

In addition,

$$
\begin{gathered}
\mathcal{L}\left(\frac{(R-\Delta)(1-k-\bar{\ell})[f(a)-g(k, \bar{\ell})](1-\tau R)}{[\bar{\ell}+\tau R(1-k-\bar{\ell})]}\right)=\lambda \mathcal{L}\left(\frac{[f(a)-g(k, \bar{\ell})]}{[\bar{\ell}+\tau R(1-k-\bar{\ell})]}\right) \\
=\lambda \mathcal{L}\left(\frac{f(a)(1-k-\bar{\ell})-k}{\bar{\ell}+\tau R(1-k-\bar{\ell})}\right)<0
\end{gathered}
$$

where $\lambda=(R-\Delta)(1-k-\bar{\ell})(1-\tau R)>0$ Therefore, $\mathcal{L}\left(T_{3}\right)<0$.

This implies that $\mathcal{L}\left(T_{i}\right)<0$ for $1 \leq i \leq 4$, and $\mathcal{L}\left(T_{\text {solv }}\right)<0$.

Furthermore,

$$
\mathcal{L}\left(f_{0}\right)=\mathcal{L}\left(\frac{R(1-k-\bar{\ell})+k}{(R+1)(1-k-\bar{\ell})}\right)>0 \Longrightarrow \mathcal{L}\left(a_{0}(k, \bar{\ell})\right)<0
$$

Therefore,

$$
\mathcal{L}\left(a_{0}\right)\left[T_{1}\left(a_{0}, k, \bar{\ell}\right)+T_{2}\left(a_{0}, k, \bar{\ell}\right) \mathbb{1}_{\underline{m}=a_{0}}+T_{3}\left(a_{0}, k, \bar{\ell}\right) \mathbb{1}_{\underline{m}<a_{0} \text { and } m_{1}=a_{0}}+T_{4}\left(a_{0}, k, \bar{\ell}\right) \mathbb{1}_{\underline{m}, m_{1}<a_{0}}-T_{\text {solv }}\left(a_{0}, k, \bar{\ell}\right)\right]<0
$$

Together, this result, along with the fact that $\mathcal{L}\left(T_{i}\right)<0$ for $1 \leq i \leq 4$, and $\mathcal{L}\left(T_{\text {solv }}\right)<0$ means we can prove the result by Leibniz's Rule, i.e., $\mathcal{L}\left(\alpha_{1}(k, \bar{\ell})\right)<0$.

Proof of part c. Let us denote $\mathbb{E} \Pi(a, b, c)$ to be the expected profit for a given bank that carries liquidity $b$ when the margin is $a$ and all other banks carry liquidity $c$.

Claim: Let $k$ and $\bar{\ell}$ be such that both $(k, \bar{\ell})$ and $(k+\bar{\ell}, 0)$ are possible equilibria in the context of the definition in Section 4. Then,

$$
\mathbb{E} \Pi(k, \ell, \bar{\ell})<\mathbb{E} \Pi(k+\bar{\ell}, 0,0)
$$

i.e., it is always optimal for banks to not carry over any liquidity and only risk-share in the form of the margin. 
Proof. Recall from Section 3 that

$$
\begin{aligned}
\mathbb{E} \Pi(k, \ell, \bar{\ell})= & \\
& \int_{-\infty}^{a_{0}(k, \bar{\ell})}[R(1-k-\ell)+\ell](1-f(a)+g(k, \bar{\ell})) \phi(a) d a \\
& +\int_{-\infty}^{\min \left(a, a_{0}\right)}[(R-\Delta-1) y(0, k, \ell)(1-f(a))+\ell(f(a)-g(k, \bar{\ell}))] \phi(a) d a \\
& +\int_{\min \left(a_{0}, a_{1}\right)}^{\min \left(a_{0}\right)}[(R-\Delta-1-p) y(p, k, \ell)(1-f(a))+[p(1-k-\ell)+\ell](f(a)-g)] \phi(a) d a \\
& +\int_{\min \left(a_{0}, a_{0}, a_{1}\right)}^{a^{\prime}}[(R-\Delta-1)(1-k-\ell)+\ell)(f(a)-g(k, \bar{\ell})] \phi(a) d a \\
& +\int_{a_{0}(k, \bar{\ell})}^{\infty}([R(1-k-\ell)(1-\eta(f(a)))(1-f(a))+R(1-k-\ell) f(a)]) \phi(a) d a
\end{aligned}
$$

Now, let us assume that $(k, \bar{\ell})$ is an equilibrium. Then, by definition, $\mathbb{E} \Pi(k, \ell, \bar{\ell})=\mathbb{E} \Pi(k, \bar{\ell}, \bar{\ell})$ We have that

$$
\begin{aligned}
& \begin{array}{r}
(R-\Delta- \\
=
\end{array} \begin{array}{r}
p(k, \bar{\ell})) y(p, k, \bar{\ell})(1-f(a))+p(k, \bar{\ell})(1-k-\bar{\ell})(f(a)-g(k, \bar{\ell})) \\
=(R-\Delta) \frac{[f(a)-g(k, \bar{\ell})]}{1-f(a)}(1-k-\bar{\ell})(1-f(a))-(1+p(k, \bar{\ell})) y(p, k, \bar{\ell})(1-f(a)) \\
\quad+(1+p(k, \bar{\ell}))(1-k-\bar{\ell})(f(a)-g(k, \bar{\ell}))-(1-k-\bar{\ell})(f(a)-g(k, \bar{\ell})) \\
=(R-\Delta)[f(a)-g(k, \bar{\ell})](1-k-\bar{\ell})-(1-f(a))[\bar{\ell}+\tau R(1-k-\bar{\ell})]+(1-f(a))[\bar{\ell}+\tau R(1-k-\bar{\ell})] \\
-(1-k-\bar{\ell})[f(a)-g(k, \bar{\ell})] \\
=(R-\Delta-1)[f(a)-g(k, \bar{\ell})](1-k-\bar{\ell})=(R-\Delta-1) f(a)(1-k-\bar{\ell})-k(R-\Delta-1)
\end{array}
\end{aligned}
$$

where the third step follows from the fact that $(1+p(k, \bar{\ell})) y(p, k, \bar{\ell})=\bar{\ell}+\tau R(1-k-\bar{\ell})$. Plugging this in to the expression for profit, we get that

$$
\begin{aligned}
\mathbb{E} \Pi(k, \bar{\ell}, \bar{\ell})= & \int_{-\infty}^{a_{0}(k, \bar{\ell})}[R(1-k-\bar{\ell})+\bar{\ell}](1-f(a)+g(k, \bar{\ell})) \phi(a) d a \\
& +\int_{-\infty}^{\min \left(\underline{a}, a_{0}\right)}((R-\Delta-1)[\bar{\ell}+\tau R(1-k-\bar{\ell})](1-f(a))+\bar{\ell}[f(a)-g(k, \bar{\ell})]) \phi(a) d a \\
& +\int_{\min \left(\underline{a}, a_{0}\right)}^{\min \left(a_{0}, a_{1}\right)}((R-\Delta-1)[f(a)-g(k, \bar{\ell})](1-k-\bar{\ell})+\bar{\ell}[f(a)-g(k, \bar{\ell})]) \phi(a) d a \\
& +\int_{\min \left(a_{0}, a_{1}\right)}^{a_{0}(k, \bar{\ell})}[(R-\Delta-1)(1-k-\bar{\ell})+\bar{\ell})(f(a)-g(k, \bar{\ell})] \phi(a) d a \\
& +\int_{a_{0}(k, \bar{\ell})}^{\infty}\left(R(1-k-\bar{\ell})\left[1-\frac{f(a)}{R}+\frac{k}{R(1-k-\bar{\ell})}\right]\right) \phi(a) d a
\end{aligned}
$$

From now on, let us denote $\mathbb{E} \Pi(k, \bar{\ell}, \bar{\ell})$ by $\mathbb{E} \Pi(k, \bar{\ell})$.

To prove the proposition, let us first calculate $\partial \mathbb{E} \Pi(k, \bar{\ell}) /\left.\partial k\right|_{k+\bar{\ell}=c}$. This is the derivative of the profit function with respect to $k$, keeping $k+\bar{\ell}$ fixed (and equal to $c$. Put another way, this equals $\partial \mathbb{E} \Pi(k, \bar{\ell}) / \partial k-\partial \mathbb{E} \Pi(k, \bar{\ell}) / \partial \bar{\ell}$.

Now, $\partial \mathbb{E} \Pi(k, \bar{\ell}) /\left.\partial k\right|_{k+\bar{\ell}=c}$ If this is positive, then clearly $\mathbb{E} \Pi(k+\bar{\ell}, 0)>\mathbb{E} \Pi(k, \bar{\ell})$. Note that $\mathbb{E} \Pi(k, \bar{\ell})$ is a sum of different integrals, where the limits of integration themselves depend on $k$ and $\bar{\ell}$. Then, by the Leibniz Rule, we have that $\partial \mathbb{E} \Pi(k, \bar{\ell}) /\left.\partial k\right|_{k+\bar{\ell}=c}=k_{1}+k_{2}$, where $k_{1}$ is the integral of the derivative of the functions inside the integrals, keeping the limits of integration fixed. $k_{2}$ is the derivative of the integral limits, multiplied by the function value at those limits. 
Noting that $g(k, \bar{\ell})=k /(1-k-\bar{\ell})$, we have that

$$
\begin{aligned}
k_{1}=\int_{-\infty}^{a_{0}} R \phi(a) d a+\int_{-\infty}^{\min \left(\underline{a}, a_{0}\right)}-(R-\Delta-1)(1- & f(a)) \phi(a) d a+\int_{\min \left(\underline{a}, a_{0}\right)}^{\min \left(a_{0}, a_{1}\right)}-(R-\Delta-1) \phi(a) d a \\
& +\int_{\min \left(a_{0}, a_{1}\right)}^{a_{0}}-(R-\Delta-1) \phi(a) d a-\int_{-\infty}^{a_{0}} \phi(a) d a
\end{aligned}
$$

Simplifying, this implies that

$$
\begin{aligned}
k_{1}=\int_{-\infty}^{a_{0}}(R-1) \phi(a) d a-(R-\Delta-1) & \int_{-\infty}^{a_{0}} \phi(a) d a+(R-\Delta-1) \int_{-\infty}^{\min \left(\underline{a}, a_{0}\right)} f(a) \phi(a) d a \\
& =\Delta \int_{-\infty}^{a_{0}} \phi(a) d a+(R-\Delta-1) \int_{-\infty}^{\min \left(\underline{a}, a_{0}\right)} f(a) \phi(a) d a>0
\end{aligned}
$$

Now, let us assume that $(R-\Delta)>1$, and let us define the operator $\mathcal{L}(f(k, \ell))=\partial f / \partial k-\partial k / \partial \ell$.

In addition, let $m_{0}=\min \left(\underline{a}, a_{0}\right)$ and $m_{1}=\min \left(a_{0}, a_{1}\right)$.

Then, by the Leibniz Rule, $k_{2}$ is given by

$$
\begin{aligned}
& k_{2}=\mathcal{L}\left(a_{0}\right)[R(1-k-\bar{\ell})+\bar{\ell}]\left(1-f\left(a_{0}\right)+g(k, \bar{\ell})\right) \phi\left(a_{0}\right)+\mathcal{L}\left(m_{0}\right)(R-\Delta-1)[\bar{\ell}+\tau R(1-k-\bar{\ell})]\left(1-f\left(m_{0}\right)\right) \phi\left(m_{0}\right) \\
& +(R-\Delta-1) \mathcal{L}\left(m_{1}\right)\left(\left[f\left(m_{1}\right)-g(k, \bar{\ell})\right](1-k-\bar{\ell})+\bar{\ell}\left[f\left(m_{1}\right)-g(k, \bar{\ell})\right]\right) \phi\left(m_{1}\right) \\
& -(R-\Delta-1) \mathcal{L}\left(m_{0}\right)\left(\left[f\left(m_{0}\right)-g(k, \bar{\ell})\right](1-k-\bar{\ell})+\bar{\ell}\left[f\left(m_{0}\right)-g(k, \bar{\ell})\right]\right) \phi\left(m_{0}\right) \\
& +[(R-\Delta-1)(1-k-\bar{\ell})+\bar{\ell}]\left[\mathcal{L}\left(a_{0}\right)\left(f\left(a_{0}\right)-g(k, \bar{\ell})\right) \phi\left(a_{0}\right)-\mathcal{L}\left(m_{1}\right)\left(f\left(m_{1}\right)-g(k, \bar{\ell})\right) \phi\left(m_{1}\right)\right] \\
& -\mathcal{L}\left(a_{0}\right)\left(R(1-k-\bar{\ell})\left[1-\frac{f\left(a_{0}\right)}{R}+\frac{k}{R(1-k-\bar{\ell})}\right]+\bar{\ell}\right) \phi\left(a_{0}\right)
\end{aligned}
$$

After some algebra, this becomes

$$
\begin{aligned}
& k_{2}=\mathcal{L}\left(a_{0}\right)[R(1-k-\bar{\ell})+\bar{\ell}]\left(1-f\left(a_{0}\right)+g(k, \bar{\ell})\right) \phi\left(a_{0}\right)+\mathcal{L}\left(m_{0}\right)(R-\Delta-1)[\bar{\ell}+\tau R(1-k-\bar{\ell})]\left(1-f\left(m_{0}\right)\right) \phi\left(m_{0}\right) \\
& +[(R-\Delta-1)(1-k-\bar{\ell})+\bar{\ell}]\left[\mathcal{L}\left(a_{0}\right)\left(f\left(a_{0}\right)-g(k, \bar{\ell})\right) \phi\left(a_{0}\right)-\mathcal{L}\left(m_{0}\right)\left(f\left(m_{0}\right)-g(k, \bar{\ell})\right) \phi\left(m_{1}\right)\right] \\
& -\mathcal{L}\left(a_{0}\right)\left(R(1-k-\bar{\ell})\left[1-\frac{f\left(a_{0}\right)}{R}+\frac{k}{R(1-k-\bar{\ell})}\right]+\bar{\ell}\right) \phi\left(a_{0}\right)
\end{aligned}
$$

and then

$$
\begin{gathered}
k_{2}=\mathcal{L}\left(a_{0}\right)\left([R(1-k-\bar{\ell})+\bar{\ell}]\left(1-f\left(a_{0}\right)+g(k, \bar{\ell})\right)+[(R-\Delta-1)(1-k-\bar{\ell})+\bar{\ell}]\left(f\left(a_{0}\right)-g(k, \bar{\ell})\right)\right. \\
\left.-R(1-k-\bar{\ell})\left[1-\frac{f\left(a_{0}\right)}{R}+\frac{k}{R(1-k-\bar{\ell})}\right]+\bar{\ell}\right) \phi\left(a_{0}\right) \\
\left.+\mathcal{L}\left(m_{0}\right)\left((R-\Delta-1)[\bar{\ell}+\tau R(1-k-\bar{\ell})]\left(1-f\left(m_{0}\right)\right)-(1-k-\bar{\ell})\left(f\left(m_{0}\right)-g(k, \bar{\ell})\right)\right]\right) \phi\left(m_{1}\right) \quad(\mathrm{A} 13)
\end{gathered}
$$

Lemma A1. $\mathcal{L}\left(m_{0}\right) ; \mathcal{L}\left(a_{0}\right)<0$

Proof. First, recall that $\underline{f}, f_{0}$ and $f_{1}$ are defined such that $f>\underline{f}$ whenever $a<\underline{a}$ and so on. Then, from earlier work,

$$
\begin{gathered}
f_{1}(k, \ell)=\frac{(\bar{\ell}+\tau R(1-k-\ell))+k(R-\Delta)}{\bar{\ell}+\tau R(1-k-\bar{\ell})+(1-k-\bar{\ell})(R-\Delta)} ; \underline{f}(k, \ell)=\frac{k+\bar{\ell}+\tau R(1-k-\ell \overline{)})}{(1-k-\bar{\ell})+(\bar{\ell}+\tau R(1-k-\bar{\ell}))} \\
f_{0}(k, \ell)=\frac{k+R(1-k-\bar{\ell})}{(1+R)(1-k-\bar{\ell})}
\end{gathered}
$$

Then,

$$
\mathcal{L}(\underline{f})=\frac{\underline{f}}{(1-k)+\tau R(1-k-\bar{\ell})} ; \mathcal{L}\left(f_{1}\right)=\frac{f_{1}+(R-\Delta-1)}{\bar{\ell}+\tau R(1-k-\bar{\ell})+(1-k-\bar{\ell})(R-\Delta)}
$$




$$
\mathcal{L}\left(f_{0}\right)=\frac{1}{(1+R)(1-k-\bar{\ell})}
$$

In general, for any aggregate shock $a$, the number of failures $f(a)$ is given by $f(a)=\Phi((c-$ $\sqrt{\rho} a) / \sqrt{1-\rho}$. Therefore,

$$
\mathcal{L}(a)=-\frac{\sqrt{1-\rho}}{\sqrt{\rho}} \times \frac{1}{\phi\left(N^{-1} f(a)\right)} \times \mathcal{L}(f)
$$

and the result follows since $\mathcal{L}(f)>0$ for $f=f, f_{0}$, and $f\left(a_{0}\right)>R /(R+1)>1 / 2$.

Now, note that for any $k, \bar{\ell}$,

$$
\begin{array}{r}
{[R(1-k-\bar{\ell})+\bar{\ell})\left(1-f\left(a_{0}\right)+g(k, \bar{\ell})\right)+(R-\Delta-1)(1-k-\bar{\ell})\left(f\left(a_{0}\right)-g(k, \bar{\ell})\right)+\bar{\ell}\left(f\left(a_{0}\right)-g(k, \bar{\ell})\right)} \\
=R(1-k-\bar{\ell}) f\left(a_{0}\right)+\bar{\ell}
\end{array}
$$

In addition, $f\left(a_{0}\right)$ satisfies $f\left(a_{0}\right)=\left[1-f\left(a_{0}\right) / R+k / R(1-k-\bar{\ell})\right]$

Therefore, Equation (1) implies that

$$
\left.k_{2}=\mathcal{L}\left(m_{0}\right)\left((R-\Delta-1)[\bar{\ell}+\tau R(1-k-\bar{\ell})]\left(1-f\left(m_{0}\right)\right)-(1-k-\bar{\ell})\left(f\left(m_{0}\right)-g(k, \bar{\ell})\right)\right]\right) \phi\left(m_{1}\right)
$$

Since we know that $\mathcal{L}\left(m_{0}\right)<0$ and $\phi\left(m_{0}\right)>0$, we just have to show that

$$
[\bar{\ell}+\tau R(1-k-\bar{\ell})]\left(1-f\left(m_{0}\right)\right) \leq(1-k-\bar{\ell})\left(f\left(m_{0}\right)-g(k, \bar{\ell})\right)
$$

Since $g(k, \bar{\ell})=k /(1-k-\bar{\ell})$, and doing some algebra, we must show that

$$
f\left(m_{0}\right) \geq \frac{k+\bar{\ell}+\tau R(1-k-\bar{\ell})}{1-k+\tau R(1-k-\bar{\ell})} \text { i.e., } f\left(m_{0}\right) \geq \underline{f}
$$

However, recall that $m_{0}=\min \left(a_{0}, \underline{a}\right)$. This means that $m_{0} \leq \underline{a}$, and so $f\left(m_{0}\right) \geq \underline{f}$.

\section{Appendix D. Proof of Proposition 4}

We first derive the expected profit for banks if there is no clearinghouse, and banks carry liquidity $\ell$. If $\ell \geq 1 / 2$, banks self hedge and refinance themselves if required. There is no fire sale. If all banks carry liquidity $\ell$ (determined in equilibrium), then a fire sale might well occur. Let us restrict our attention to the case where all banks carry liquidity $\bar{\ell}<1 / 2$, and derive the profit for a bank carrying liquidity $\ell<1 / 2$. Then,

$$
\begin{aligned}
\mathbb{E} \Pi(\ell, \bar{\ell})= & \\
& \int_{-\infty}^{\infty}[R(1-\ell)+\ell](1-f(a)) \phi(a) d a \\
& +\int_{-\infty}^{\underline{a}}[(R-\Delta-1) y(0, \ell)(1-f(a))+\ell(f(a))] \phi(a) d a \\
& \left.+\int_{\underline{a}}^{a_{1}}[(R-\Delta-1-p \bar{\ell})) y(p, \ell)(1-f(a))+[p(\bar{\ell}) \times(1-\ell)+\ell] f(a)\right] \phi(a) d a \\
& +\int_{a_{1}}^{\infty}[((R-\Delta-1)(1-\ell)+\ell) f(a)] \phi(a) d a
\end{aligned}
$$

If $\bar{\ell}$ is an equilibrium, then for any $\ell, \mathbb{E} \Pi(\ell, \bar{\ell})=\mathbb{E} \Pi(\bar{\ell}, \bar{\ell})$. We can substitute $\ell=\bar{\ell}$ in the equation above to get 


$$
\begin{aligned}
\mathbb{E} \Pi(\bar{\ell}, \bar{\ell})= & \int_{-\infty}^{\infty}[R(1-\bar{\ell})+\bar{\ell}](1-f(a)) \phi(a) d a \\
& +\int_{-\infty}^{\underline{a}}((R-\Delta-1)[\bar{\ell}+\tau R(1-\bar{\ell})](1-f(a))+\bar{\ell} f(a)) \phi(a) d a \\
& +\int_{\underline{a}}^{a_{1}}((R-\Delta-1) f(a)(1-\bar{\ell})+\bar{\ell} f(a)) \phi(a) d a \\
& +\int_{a_{1}}^{\infty}[(R-\Delta-1)(1-\bar{\ell})+\bar{\ell}] f(a) \phi(a) d a
\end{aligned}
$$

The integrand in the third integral is lower than the one in the fourth integral since $f(a)=1$. In addition, the integrand in the second integral is a special case of the one in the third integral with $p(\bar{\ell})$ set to zero. Therefore, the integrands in the second and third integrals in the expression above are both lower than the integrand in the fourth integral.

So, we have that

$$
\begin{aligned}
\mathbb{E} \Pi(\bar{\ell}, \bar{\ell}) \leq & \int_{-\infty}^{\infty}[R(1-\bar{\ell})+\bar{\ell}](1-f(a)) \phi(a) d a \\
& \left.+\int_{-\infty}^{\infty}[(R-\Delta-1)(1-\bar{\ell})+\bar{\ell})\right] f(a) \phi(a) d a
\end{aligned}
$$

Simplifying the above, we get that

$$
\mathbb{E} \Pi(\bar{\ell}, \bar{\ell}) \leq \bar{\ell}+(1-\bar{\ell})(R-\alpha)<(1+R-\alpha) / 2
$$

where we used the fact that $\int_{-\infty}^{\infty} f(a) d a=\alpha$.

This completes the proof of the proposition.

\section{Appendix E. Proof of Proposition 5}

The proof is immediate using the results already obtained in earlier proofs. In particular, note that

$$
\mathbb{E} \Pi^{F B}(k, \bar{\ell})=\mathbb{E} \Pi(k, \bar{\ell}, \bar{\ell})
$$

We have already shown that $\mathbb{E} \Pi(k, \bar{\ell}, \bar{\ell})<\mathbb{E} \Pi(k+\bar{\ell}, 0,0)$. Therefore, it becomes clear that $\ell^{F B}=0$.

However, conditioning on $\ell^{F B}=0$, the first-best problem becomes formally identical to the equilibrium problem when $\ell=\bar{\ell}=0$, which we know to be true at the optimal equilibrium $k^{*}$. Therefore, $k^{F B}=k^{*}$.

Since margins and liquidity are identical under the first-best measure as under equilibrium, there is similar equivalence of profits and systemic risk.

This completes the proof of the proposition.

\section{Appendix F. Proof of Proposition 6}

$$
\begin{aligned}
& \text { Let } \mathcal{C}(k, \bar{\ell})=\Phi\left(a_{g}(k, \bar{\ell})\right) c\left(g^{*}\right)+\int_{a_{g}(k, \bar{\ell})}^{a_{0}(k, \bar{\ell})} a_{g o v}\left[f(a)-f_{0}\right]^{2}(1-k-\bar{\ell})^{2} \text { Then, } \\
& \frac{d \mathcal{C}(k, \bar{\ell})}{d k}=c\left(g^{*}\right) \phi\left(a_{g}\right) \frac{\partial a_{g}}{\partial k}+2 \int_{a_{g}}^{a_{0}}(1-k-\bar{\ell}) a_{g o v}\left[f\left(a_{g}\right)-f_{0}\right]^{2}+\frac{\partial a_{g}}{\partial k} a_{g o v}\left[f\left(a_{g}\right)-f_{0}\right]^{2}(1-k-\bar{\ell})^{2}<0
\end{aligned}
$$

Therefore, $d W(k, \bar{\ell}(k)) / d k=d \mathbb{E} \Pi(k, \bar{\ell}(k)) / d k-d \mathcal{C}(k, \bar{\ell}(k)) / d k>d \mathbb{E} \Pi(k, \bar{\ell}(k)) / d k$ 
In addition, as $k \rightarrow 1 / 2, a_{g} \rightarrow a_{0}$ and $\mathcal{C}(k, \bar{\ell}(k)) \rightarrow 0$.

Now, let $k^{*}$ solve $k^{*}=\arg \max \mathbb{E} \Pi(k, \bar{\ell}(k))$. Then, for $k<k^{*}, \mathbb{E} \Pi\left(k, \bar{\ell}(k)<\mathbb{E} \Pi\left(k^{*}, \bar{\ell}\left(k^{*}\right)\right.\right.$, and so, since $\mathcal{C}(k, \bar{\ell}(k))>0$, and $d \mathcal{C}(k, \bar{\ell}(k)) / d k<0$, we must have that $W\left(k, \bar{\ell}(k)<W\left(k^{*}, \bar{\ell}\left(k^{*}\right)\right.\right.$. Since, by definition, $k_{\text {pvt }}=k^{*}$, this means that $k_{\text {pub }} \geq k_{\text {pvt }}$, completing the proof.

\section{Appendix G. Note on Asymmetric Equilibria}

Let us now relax our assumption that all banks carry the same liquidity $\bar{\ell}$ and instead assume that there is an asymmetric equilibrium where a fraction $w_{1}$ of the banks carry over liquidity $\bar{\ell}_{1}$, while a fraction $w_{2}=\left(1-w_{1}\right)$ of the banks carry over liquidity $\bar{\ell}_{2}$.

As before, a fraction $\tau$ of future payoffs can be pledged by banks in the fire sale, so the fire sale demand function for a bank with liquidity $\ell$ is given by

$$
y(p, k, \ell)=\frac{\ell+\tau R(1-k-\ell)-y(p, k, \ell)}{p}
$$

where $p$ is the price of assets in the fire sale.

For convenience, let us refer to banks that carry liquidity $\bar{\ell}_{1}$ as "Type 1 banks", and banks that carry liquidity $\bar{\ell}_{2}$ as "Type 2 banks". Let $\bar{y}_{1}$ and $\bar{y}_{2}$ be the total demand submitted by Type 1 banks and Type 2 banks respectively. Then,

$$
\bar{y}_{1}=\frac{\bar{\ell}_{1}+\tau R\left(1-k-\bar{\ell}_{1}\right)-\bar{y}_{1}}{p} ; \bar{y}_{2}=\frac{\bar{\ell}_{2}+\tau R\left(1-k-\bar{\ell}_{2}\right)-\bar{y}_{2}}{p}
$$

As before, let $g$ be the total number of banks bailed out by the clearinghouse (with margin $k$ ) if it is insolvent. The clearinghouse bails out the failed banks with its margin, using a total amount of $w_{1} k$ to bail out Type 1 banks, and $w_{2} k$ to bail out Type 2 banks. Then the total number of banks bailed out by the clearinghouse is given by

$$
g\left(k, w_{1}, w_{2}, \bar{\ell}_{1}, \bar{\ell}_{2}\right)=\frac{w_{1} k}{\left(1-k-\bar{\ell}_{1}\right)}+\frac{w_{2} k}{\left(1-k-\bar{\ell}_{2}\right)}
$$

Let $g_{1}=w_{1} k /\left(1-k-\bar{\ell}_{1}\right)$ and $g_{2}=w_{2} k /\left(1-k-\bar{\ell}_{2}\right)$ denote the number of banks of Type 1 and Type 2 bailed out.

Then, the supply of assets in the fire sale is given by

$$
S=\left(1-k-\bar{\ell}_{1}\right)\left[w_{1} f(a)-g_{1}\right]+\left(1-k-\bar{\ell}_{2}\right)\left[w_{2} f(a)-g_{2}\right]
$$

The demand of assets in the fire sale is given by

$$
D=w_{1}(1-f(a)) \bar{y}_{1}+w_{2}(1-f(a)) \bar{y}_{2}
$$

where $w_{1}(1-f(a))$ is the number of banks that survived that are of Type 1 , while $w_{2}(1-f(a))$ is the number of banks that survived that are of Type 2.

Therefore, the market clearing condition is

$$
w_{1} \bar{y}_{1}+w_{2} \bar{y}_{2}=\frac{\left(1-k-\bar{\ell}_{1}\right)\left[w_{1} f(a)-g_{1}\right]+\left(1-k-\bar{\ell}_{2}\right)\left[w_{2} f(a)-g_{2}\right]}{1-f(a)}
$$

Further, Equation (A14) implies that

$$
p\left(k, w_{1}, w_{2}, \bar{\ell}_{1}, \bar{\ell}_{2}\right)=-1+\frac{\bar{\ell}_{1}+\tau R\left(1-k-\bar{\ell}_{1}\right)}{\bar{y}_{1}}=-1+\frac{\bar{\ell}_{2}+\tau R\left(1-k-\bar{\ell}_{2}\right)}{\bar{y}_{2}}
$$


Equation (A15) implies that

$$
\bar{y}_{1} w_{1}(1-f)+\bar{y}_{2} w_{2}(1-f)-f(1-k)+w_{1} f \bar{\ell}_{1}+w_{2} f \bar{\ell}_{2}+k=0
$$

Together, Equations (A15) and (A16) imply values for $\bar{y}_{1}$ and $\bar{y}_{2}$.

$$
\bar{y}_{2}=\frac{f(1-k)-k-f\left[w_{1} \bar{\ell}_{1}+w_{2} \bar{\ell}_{2}\right]}{(1-f)\left[\eta w_{1}+w_{2}\right]} ; \bar{y}_{1}=\frac{f(1-k)-k-f\left[w_{1} \bar{\ell}_{1}+w_{2} \bar{\ell}_{2}\right]}{(1-f)\left[w_{1}+w_{2} / \eta\right]}
$$

where $\bar{y}_{1}=\eta \bar{y}_{2}$ and

$$
\eta=\frac{\bar{\ell}_{1}+\tau R\left(1-k-\bar{\ell}_{1}\right)}{\bar{\ell}_{2}+\tau R\left(1-k-\bar{\ell}_{2}\right)}
$$

Defining thresholds:

As before, let $\eta(f)$ denote the portion of payoffs that successful banks transfer to failed banks if the clearinghouse remain solvent. Then,

$$
\eta(f)=\frac{w_{1} f\left(1-k-\bar{\ell}_{1}\right)+w_{2} f\left(1-k-\bar{\ell}_{2}\right)-k}{R\left(1-k-\bar{\ell}_{1}\right)(1-f) w_{1}+R\left(1-k-\bar{\ell}_{2}\right)(1-f) w_{2}}
$$

If $f$ is such that $\eta(f)>1$, then the clearinghouse becomes insolvent. Let $f^{*}$ denote this unique threshold, and $a_{0}$ denote the level of aggregate shock $a$ at which $f=f^{*}$.

As before, let $f_{1}$ denote the number of failures above which there is a fire sale with price less than $(R-\Delta-1) \cdot f_{1}$ is determined from Equation (A16). Let $a_{1}$ denote the level of aggregate shock $a$ at which $f=f_{1}$.

Expected profits are given by

$$
\begin{aligned}
\mathbb{E} \Pi\left(k, \ell, \bar{\ell}_{1}, \bar{\ell}_{2}\right)= & \\
& \int_{-\infty}^{a_{0}\left(k, \bar{\ell}_{1}, \bar{\ell}_{2}\right)}[R(1-k-\ell)+\ell]\left(1-f(a)+g_{1}+g_{2}\right) \phi(a) d a \\
& +\int_{-\infty}^{\min \left(a_{0}, a_{1}\right)}[(R-\Delta-1-p) y(p, k, \ell)(1-f(a))+[p(1-k-\ell)+\ell](f(a)-g)] \phi(a) d a \\
& \left.+\int_{\left.\min \left(k, l_{0}\right), a_{1}\right)}^{a_{0}}[(R-\Delta-1)(1-k-\ell)+\ell)\left(f(a)-g_{1}-g_{2}\right)\right] \phi(a) d a \\
& +\int_{a_{0}(k, \bar{\ell})}^{\infty}([R(1-k-\ell)(1-\eta(f(a)))(1-f(a))+R(1-k-\ell) f(a)]+\ell) \phi(a) d a
\end{aligned}
$$

Bailing out Firms in the Asymmetric Equilibrium Model

One issue that becomes important when writing out the asymmetric equilibrium model is how failed banks are bailed out with the margin available in the clearinghouse $(k)$. If $k_{1}$ of the margin is used to bail out Type 1 banks and $k_{2}$ of the margin is used to bail out Type 2 banks, then the total number of banks bailed out is given by

$$
g\left(k_{1}, k_{2}, \bar{\ell}_{1}, \bar{\ell}_{2}\right)=\frac{k_{1}}{1-k-\bar{\ell}_{1}}+\frac{k_{2}}{1-k-\bar{\ell}_{2}}
$$

Let $g_{1}$ and $g_{2}$ be the proportion of Type 1 and Type 2 banks (respt.) bailed out. Then,

$$
g_{1}=\frac{k_{1}}{1-k-\bar{\ell}_{1}} ; g_{2}=\frac{k_{2}}{1-k-\bar{\ell}_{2}}
$$

The supply of assets in the fire sale is given by

$$
\mathcal{S}=\left(1-k-\bar{\ell}_{1}\right)\left[w_{1} f(a)-g_{1}\right]+\left(1-k-\bar{\ell}_{2}\right)\left[w_{2} f(a)-g_{2}\right]=\left(1-k-\bar{\ell}_{1}\right) \times w_{1} f(a)+\left(1-k-\bar{\ell}_{2}\right) \times w_{2} f(a)-k_{1}-k_{2}
$$

This implies that

$$
\mathcal{S}=\left(1-k-\bar{\ell}_{1}\right) \times w_{1} f(a)+\left(1-k-\bar{\ell}_{2}\right) \times w_{2} f(a)-k
$$


which is independent of $k_{1}$ and $k_{2}$. Thus the total supply of assets in the fire sale is independent of the way the clearinghouse bails out banks of each type.

Earlier, I had assumed that $k_{1}=w_{1} k$ and $k_{2}=w_{2} k$, i.e., the portion of margin used to bail out each bank type were proportional to the number of banks of each type. Instead, let

$$
k_{1}=\frac{w_{1}\left(1-k-\bar{\ell}_{1}\right) k}{w_{1}\left(1-k-\bar{\ell}_{1}\right)+w_{2}\left(1-k-\bar{\ell}_{2}\right)} ; k_{2}=\frac{w_{2}\left(1-k-\bar{\ell}_{2}\right) k}{w_{1}\left(1-k-\bar{\ell}_{1}\right)+w_{2}\left(1-k-\bar{\ell}_{2}\right)}
$$

Then,

$$
g_{1}=\frac{w_{1} k}{w_{1}\left(1-k-\bar{\ell}_{1}\right)+w_{2}\left(1-k-\bar{\ell}_{2}\right)} ; g_{2}=\frac{w_{2} k}{w_{1}\left(1-k-\bar{\ell}_{1}\right)+w_{2}\left(1-k-\bar{\ell}_{2}\right)}
$$

In the above, $g_{1} / g_{2}=w_{1} / w_{2}$, and so this is consistent with a framework where each dollar of margin in the clearinghouse is used to bail out a bank at random, whether it is a Type 1 or a Type 2 bank. The law of large numbers implies that the proportion of each type bailed out will be $w_{1}$ and $w_{2}$.

Claim: If $g_{1}$ and $g_{2}$ are defined in this way, then $\mathbb{E} \Pi\left(k, \ell, \bar{\ell}_{1}, \bar{\ell}_{2}\right)$ is a function of $w_{1} \ell_{1}+w_{2} \ell_{2}$. We can then write $\mathbb{E} \Pi\left(k, \ell, \bar{\ell}_{1}, \bar{\ell}_{2}\right)=\alpha_{0}\left(k, w_{1} \ell_{1}+w_{2} \ell_{2}\right)+\ell \alpha_{1}\left(k, w_{1} \ell_{1}+w_{2} \ell_{2}\right)$.

Further, for any asymmetric equilibrium $\left(\ell_{1}^{*}, \ell_{2}^{*}, w_{1}, w_{2}\right), \exists$ a unique symmetric equilibrium $\ell^{*}(k)$ that generates the same profits for banks.

Proof. The proof is immediate from the above result. Set $\ell^{*}(k)=w_{1} \ell_{1}^{*}+w_{2} \ell_{2}^{*}$. Then $\left(\ell^{*}, y, 1,0\right)$ is a symmetric equilibrium for any $y$, since $\alpha_{1}\left(\ell^{*}, y, 1,0\right)=0$. Further, $\alpha_{0}\left(\ell^{*}, y, 1,0\right)=\alpha_{0}\left(\ell_{1}^{*}, \ell_{2}^{*}, w_{1}, w_{2}\right)$, and so profits for the asymmetric equilibrium and the symmetric equilibrium are the same.

\section{References}

Acemoglu, Daron, and Fabrizio Zilibotti. 1997. Was Prometheus Unbound by Chance? Risk, Diversification, and Growth. Journal of Political Economy 105: 709-51. [CrossRef]

Acharya, Viral, and Alberto Bisin. 2014. Counterparty Risk Externality: Centralized versus Over-the-counter Markets. Journal of Economic Theory 149: 153-82. [CrossRef]

Acharya, Viral, and Alberto Bisin. 2009. Managerial Hedging, Equity Ownership, and Firm Value. RAND Journal of Economics 40: 47-77. [CrossRef]

Acharya, Viral, Hyun Song Shin, and Tanju Yorulmazer. 2011. Fire-sale FDI. Korean Economic Review. 27: 163-202. [CrossRef]

Acharya, Viral, Thomas Cooley, Matthew Richardson, and Ingo Walter. 2010. Manufacturing Tail Risk: A Perspective on the Financial Crisis of 2007-09. Foundations and Trends in Finance 4: 247-325. [CrossRef]

Allen, Franklin, and Elena Carletti. 2006. Credit Risk Transfer and Contagion. Journal of Monetary Economics 53: 89-111. [CrossRef]

Alessandri, Piergiorgio, and Andrew Haldane. 2009. Banking on the State. London: Bank of England.

Bhattacharya, Sudipto, and Douglas Gale. 1987. Preference shocks, Liquidity and Central Bank Policy. New Approaches to Monetary Economics 35: 69-88.

Biais, Bruno, Florian Heider, and Marie Hoerova. 2012. Clearing, Counterparty Risk and Aggregate Risk. IMF Economic Review 60: 193-222. [CrossRef]

Cassidy, John. 2009. How Markets Fail: The Logic of Economic Calamities. London: Penguin.

Castiglionesi, Fabio, Fabio Feriozzi, and Guido Lorenzoni. 2017. Financial Integration and Liquidity Crises. Management Science 65: 955-1453. [CrossRef]

Duffie, Darrell, and Haoxiang Zhu. 2011. Does a Central Clearing Counterparty Reduce Counterparty Risk? Review of Asset Pricing Studies 1: 74-95. [CrossRef]

Koeppl, Thorston, Cyril Monnet, and Ted Temzelides. 2012. Optimal Clearing Arrangements for Financial Trades. Journal of Financial Economics 103: 189-203. [CrossRef]

Leitner, Yaron. 2012. Inducing Agents to Report Hidden Trades: A Theory of an Intermediary. Review of Finance 16: 1013-42. [CrossRef]

Menkveld, Albert. 2017. Crowded Positions: An Overlooked Systemic Risk for Central Clearing Counterparties. Review of Asset Pricing Studies 7: 209-42. [CrossRef] 
Paddrik, Mark, and Simpson Zhang. 2020. Central Counterparty Default Waterfalls and Systemic Loss. Working Paper, Office of Financial Research, Washington, DC, USA.

Philippon, Thomas, and Ariell Reshef. 2012. Wages and Human Capital in the U.S. Financial Industry: 1909-2006. Quarterly Journal of Economics 127: 1551-609. [CrossRef]

Pirrong, Craig. 2009. The Economics of Clearing in Derivatives Markets: Netting, Asymmetric Information, and the Sharing of Default Risks Through a Central Counterparty. Working Paper, University of Houston, Houston, TX, USA.

Rajan, Raghuram. 2005. Has Financial Development made the World Riskier? Paper presented at a Symposium Sponsored by the Federal Reserve Bank of Kansas City, Jackson Hole, WY, USA, August 26. pp. $313-69$.

Rochet, Jean-Charles, and Guillaume Roger. 2016. Risky Utilities. Economic Theory 62: 361-82. [CrossRef]

Tucker, Paul. 2014. Are Clearing Houses the new Central Banks? Paper presented at Over-the-Counter Derivatives Symposium, Chicago, IL, USA, April 11.

Vasicek, Oldrich. 2002. The Distribution of Loan Portfolio Value. Risk 15: 160-62.

Yorulmazer, Tanju. 2013. Has Financial Innovation Made the World Riskier? CDS, Regulatory Arbitrage and Systemic Risk. Working Paper, Federal Reserve Bank of New York, New York, NY, USA.

Zawadowski, Adam. 2013. Entangled Financial Systems. Review of Financial Studies 26: 1291-323. [CrossRef]

(C) 2020 by the authors. Licensee MDPI, Basel, Switzerland. This article is an open access article distributed under the terms and conditions of the Creative Commons Attribution (CC BY) license (http://creativecommons.org/licenses/by/4.0/). 